\title{
Focal hepatic lesions in Gd-EOB-DTPA enhanced MRI: the atlas
}

\author{
José Traila Campos • Claude B. Sirlin • Jin-Young Choi
}

Received: 18 December 2011 /Revised: 15 May 2012 / Accepted: 21 May 2012 / Published online: 15 June 2012

(C) The Author(s) 2012. This article is published with open access at Springerlink.com

\begin{abstract}
Objective This article reviews the different technical aspects and pitfalls of gadolinium (Gd)-ethoxibenzyl (EOB)-diethylenetriamine pentaacetic acid (DTPA) and the advantages of the hepatocellular phase (HCP) and defines its specific imaging features of liver lesions.

Background Gd-EOB-DTPA is a contrast agent with combined properties of a conventional non-specific extracellular and a hepatocyte-specific contrast agent. Benign cirrhosisassociated nodules are characterised by isointensity in dynamic imaging and the HCP. Hepatocellular carcinomas (HCCs) usually show hyperenhancement in the arterial phase, with washout in the portal vein pressure (PVP) and hypointensity in the HCP. Among other characteristic findings, we have the mosaic pattern, a capsule, the "nodule-innodule" appearance and the satellite nodules. The fibrolamellar HCC is a large enhancing heterogeneous lesion, on a non-cirrhotic liver, with a hypointense scar in every sequence. THIDs (transient hepatic intensity differences) are perfusional alterations, characterised by hyperintensity in the arterial phase, with no alterations in the rest of the sequences including the HCP. Adenoma and focal nodular hyperplasia (FNH) are lesions, occurring more frequently in
\end{abstract}

J. T. Campos $(\bowtie)$

Department of Radiology, FMUP, Hospital S. João,

Alameda Prof. Hernâni Monteiro,

4200-319 Porto, Portugal

e-mail: jtraila@hotmail.com

\section{B. Sirlin}

Department of Radiology, UCSD,

San Diego, USA

\section{J.-Y. Choi}

Department of Radiology, Yonsei University Health System, Seoul, Republic of Korea young women, with brisk arterial enhancement, differentiated by the scar and the uptake of Gd-EOB-DTPA in the HCP. Focal eosinophilic infiltration is a difficult diagnosis, with characteristics such as a non-spherical shape and irregular borders suggesting it. Besides these lesions, in which Gd-EOB-DTPA has a clear advantage, there are a few where the specificities of this agent can be troublesome: haemangiomas, focal fat/sparing, foreign body reaction, cholangiocarcinoma and metastases.

Conclusion Gd-EOB-DTPA is comparable to extracellular agents, and uptake by functioning hepatocytes in the delayed phase provides additional information that further improves detection and characterisation of many hepatic lesions.

Main Messages

- Gd-EOB-DTPA offers advantages for the imaging of many liver lesions including HCC, fibrolamellar HCC, FNH and adenoma.

- The properties of Gd-EOB-DTPA can pose problems when dealing with haemangiomas, cholangiocarcinoma and metastases among others.

- The uptake of Gd-EOB-DTPA by functioning hepatocytes in the delayed phase provides additional information that further improves detection and characterisation of many hepatic lesions.

Keywords Magnetic resonance · MR · Gd-EOB-DTPA • Liver

\section{Introduction}

Hepatocyte-specific magnetic resonance (MR) imaging contrast agents were developed for detection and characterisation of focal liver lesions. Currently, there are two hepatocyte- 
specific agents available: gadolinium (Gd)-ethoxibenzyl (EOB)-diethylenetriamine pentaacetic acid (DTPA) and gadobenate dimeglumine (Gd-BOPTA). Gd-EOB-DTPA has greater hepatocellular uptake and biliary excretion than GdBOTPA and is the focus of this review.

Gd-EOB-DTPA - known generically as gadoxetic acid and commercially as Eovist (United States) or Primovist (most parts of the World) (Bayer Healthcare) - is a highly water-soluble contrast agent in which a lipophilic EOB group is attached to gadolinium (Gd)-DTPA (Fig. 1). The resulting compound has properties of a conventional nonspecific extracellular contrast agent in the vascular phases after administration, permitting assessment of tissue vascularity, and those of a hepatocyte-specific agent in the more delayed phases, allowing assessment of hepatocellular uptake and biliary excretion $[1,2]$. Due to the presence of the lipophilic EOB group, Gd-EOB-DTPA is actively transported from the sinusoidal space into hepatocytes via organic anion transporting polypeptides (OATPs). Entry of the contrast agent into liver cells causes intense parenchymal enhancement, beginning within 1 or 2 min of contrast agent injection. The enhancement peaks at around $20 \mathrm{~min}$ and lasts for at least $2 \mathrm{~h}$. The agent is excreted from hepatocytes into the biliary canaliculi via multidrug resistance-associated proteins (MRPs) [3, 4]. Excretion into the bile ducts causes biliary luminal enhancement, as early as $5-10 \mathrm{~min}$ after injection in normal individuals. It is eliminated in approximately equal proportions by the liver, via hepatocellular uptake with subsequent biliary excretion (43.1-53.2\%), and by the kidneys (41.6-51.2\%) [5, 6].

Gd-EOB-DTPA has been shown to be comparable to non-specific extracellular agents for lesion detection and characterisation in the vascular imaging phases. Moreover, uptake by functioning hepatocytes in the delayed phase provides additional information that further improves detection and characterisation of many hepatic lesions, reported in recent articles $[7,8]$. The purpose of this review is to illustrate the typical appearance of focal hepatic lesions with MRI and to understand the advantages, pitfalls and differential diagnosis using Gd-EOB-DTPA. The lesions are thus divided into those for which Gd-EOB-DTPA demonstrates advantages, those in which have pitfalls and those in which the lesion behaviour is equivalent to that of the use of an extracellular fluid (ECF) contrast agent.

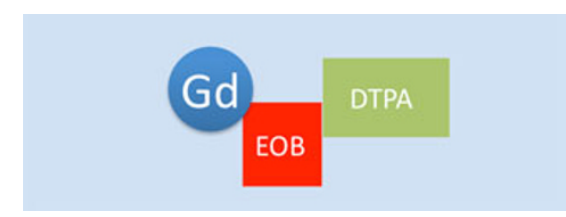

Fig. 1 Molecular structure of Gd-EOB-DTPA

\section{General considerations}

The vascular imaging phases using Gd-EOB-DTPA are similar to those using extracellular agents with a few caveats:

1. Arterial phase enhancement of vessels and lesions with Gd-EOB-DTPA tends to be less intense than with extracellular agents due to the following factors. Gd-EOBDTPA is administered in a smaller dose than extracellular agents $(0.025 \mathrm{mmol} / \mathrm{kg})$, providing a shorter temporal window to acquire images during peak arterial enhancement. Additionally, there is a higher frequency of imaging artefacts in the arterial phase when compared with extracellular agents. The cause of these artefacts is not well understood. These "truncation" artefacts may occur due to abrupt concentration changes during high spatial k-space filling, and may alter the intensity, shape and anatomical detail of the structures.

2. Hepatocellular uptake begins almost immediately, leading to rapidly progressive liver enhancement. Consequently, there is no pure venous or equilibrium phase. Because the liver enhances sooner and more intensely than with extracellular agents, the intensity of the lesions relative to the liver may appear different. For example, blood vessels and blood-pool lesions, such as haemangiomas, which remain hyperintense in comparison to the liver during all vascular phases with extracellular agents, appear to "wash out" with Gd-EOB-DTPA and, after 5-10 min, become hypointense.

3. The degree of hepatocellular enhancement depends on liver function, which roughly correlates with serum bilirubin levels, which is not a perfect predictor. In livers with good hepatic function, intense enhancement occurs. In livers without good function, due to cholestasis or hepatocellular dysfunction, enhancement of liver parenchyma may be weak. In such cases, blood vessels and blood pool lesions become isointense rather than hypointense in comparison to the liver.

\section{Focal hepatic lesions}

Lesions in which Gd-EOB-DTPA provides advantages

\section{Cirrhosis-related nodules}

1. Regenerative and dysplastic nodules

Overview: Cirrhosis is pathologically defined by extensive fibrosis, nodular regeneration and architectural distortion of the parenchyma. The vast majority of cirrhosis-related nodules exhibit regenerative changes without cellular atypia and are known as regenerative nodules (RNs). A minority have dysplastic features and 
are known as dysplastic nodules (DNs). The differentiation between RN and DN is unreliable when attempted non-invasively, difficult with histopathology, and is of minor, if any, clinical relevance. For these reasons, these two entities are discussed together as benign cirrhosisassociated nodules.

Unenhanced MRI features: Typically, benign cirrhosisassociated nodules are $<2 \mathrm{~cm}$ and isointense to hyperintense on T1-weighted images, isointense to hypointense on T2-weighted images and isointense on diffusion-weighted (DW) images. Lipid-containing nodules or steatotic nodules display signal loss on out-of-phase gradient echo (GRE) images in comparison with in-phase images. Ironcontaining nodules or siderotic nodules appear markedly hypointense on $\mathrm{T} 2$-weighted and $\mathrm{T} 2 *$-weighted images (Fig. 2).

Dynamic imaging with extracellular contrast agent: After the injection of the contrast, most benign cirrhosis associated-nodules show similar enhancement to the adjacent liver in the arterial and venous phases $[9,10]$. Some benign cirrhosis associated-nodules enhance in the arterial phase and typically fade to isointensity in the venous phases.

Imaging with $G d-E O B-D T P A$ : Uptake and excretion of gadobenate dimeglumine by these nodules is usually preserved. Consequently, they are usually isointense in the hepatocellular phase (HCP) [11].

Differential diagnosis: The diagnosis of most benign cirrhosis-associated nodules is straightforward with MRI, using extracellular agents. However, some benign cirrhosis associated-nodules may show enhancement in the arterial phase. These hypervascular benign nodules usually fade to isointensity in the venous phases. While most cirrhosisassociated nodules with arterial phase hyperenhancement and venous phase isoenhancement are benign, small hepatocellular carcinomas (HCCs) can have a similar appearance. Nodules with arterial phase hyperenhancement and venous phase isoenhancement can therefore cause diagnostic confusion and lead to inappropriate management. Use of Gd-EOB-DTPA can be helpful in the differential diagnosis of these lesions. Homogeneous isointensity in the HCP relative to liver suggests benignancy: routine follow-up is sufficient. By comparison, hypointensity in the HCP suggests malignancy: such lesions require biopsy, empirical treatment or close follow-up depending on lesion size and clinical considerations.

Potential pitfalls: Siderotic and steatotic nodules may appear hypointense in the HCP, potentially mimicking early HCC. This is due to the iron and fat content being already of low signal intensity in the pre-contrast fat saturated sequences. Some benign nodules may appear hyperintense in the HCP [14] (Fig. 3). The mechanism is still unknown, but overexpression of organic anionic transporter peptides (OATP) or down-regulation of MDRs could possibly play a role. Such lesions may be mistaken for focal nodular hyperplasia (FNH)-like lesions (these are also

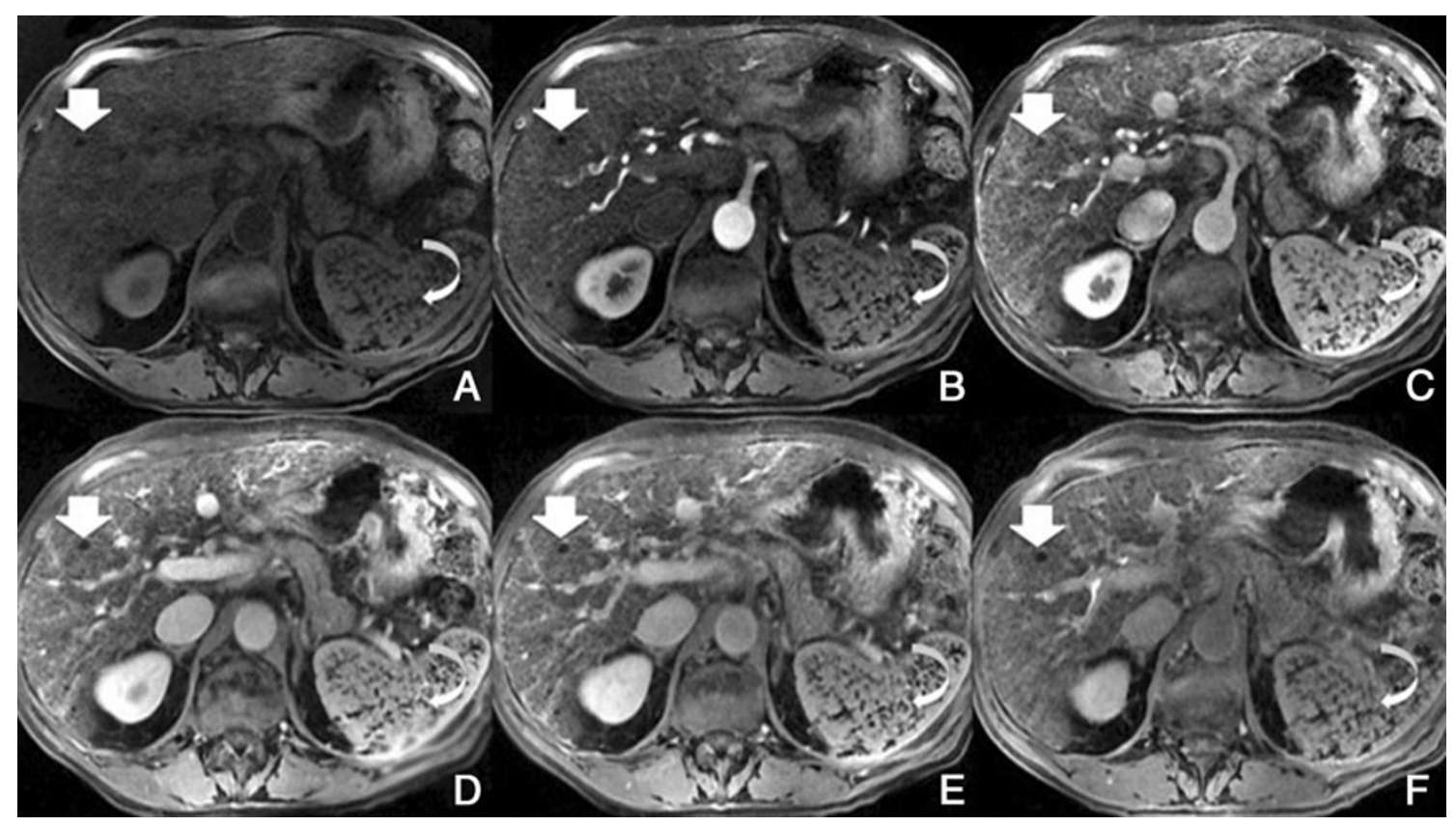

Fig. 2 A benign cirrhosis-associated nodule in a 38-year-old woman with chronic liver disease. a Pre-contrast MRI, b, $\mathbf{c}$ arterial phase, $\mathbf{d}$ PV phase, $\mathbf{e}$ delayed phase, $\mathbf{f}$ hepatocellular phase. The image shows a liver with a lobulated contour and a nodular parenchyma (cirrhosis). The solid arrows point to a small rounded focal lesion, markedly hypointense in all sequences (more conspicuous with the increase of the TE), showing no enhancement with the contrast agent. The curved arrow points to multiple Gamna-gandy bodies (hemosiderin deposition) 
Fig. 3 A benign cirrhosisassociated nodule in a 39 -yearold man with chronic liver disease. Gd-EOB-DTPA enhanced MRI. a arterial phase, b PV phase, $\mathbf{c}$ delayed phase, $\mathbf{d}$ $5 \mathrm{~min}$, e $10 \mathrm{~min}$, f hepatocellular phase. The image shows a liver with a nodular contour and a heterogeneous parenchyma. The arrows point to a small rounded focal lesion accumulating Gd-EOB-DTPA- benign nodule

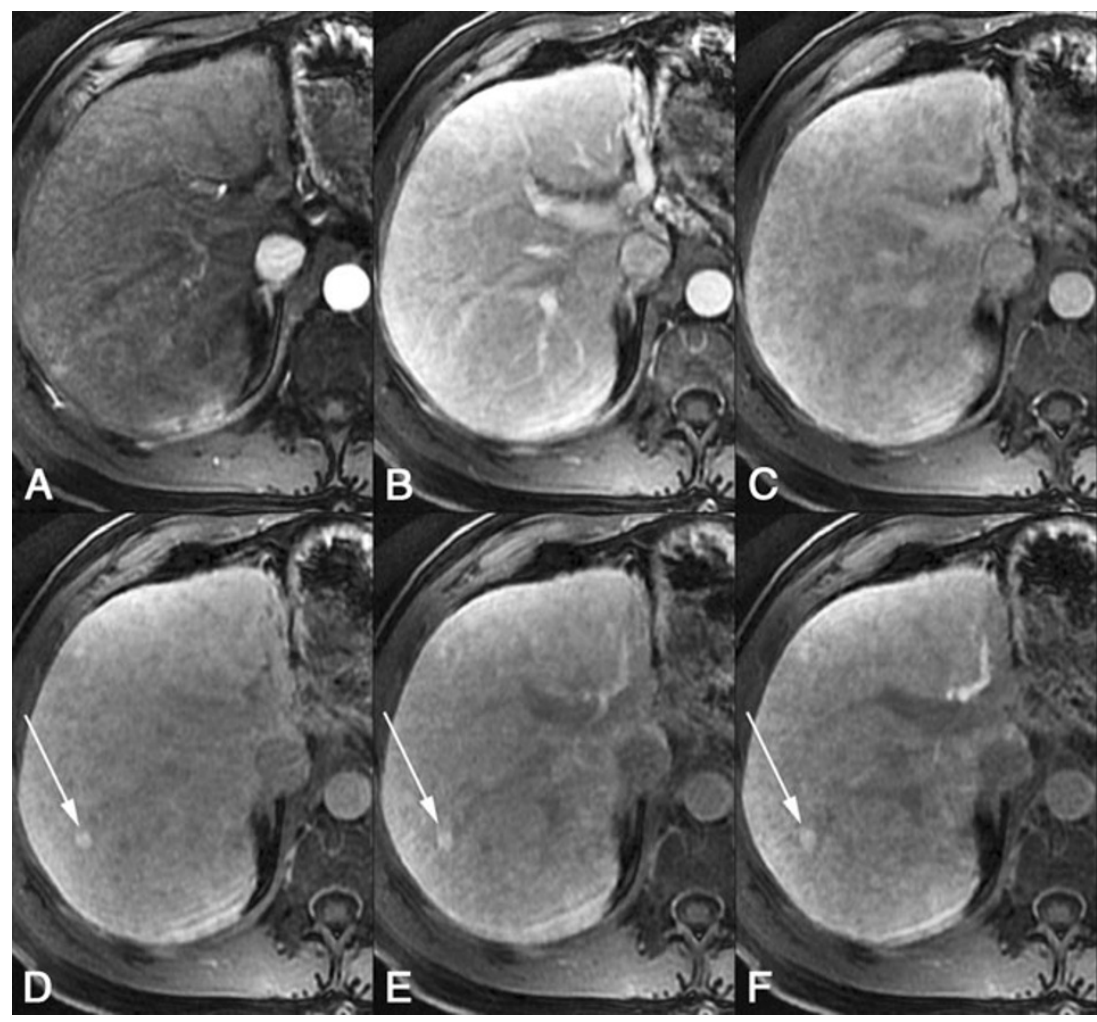

benign, so the distinction is irrelevant) or atypical HCCs with HCP hyperintensity. The differentiation between benign and malignant HCP-hyperintense nodules is discussed in the following two sections. Small benign cirrhosisassociated nodules with arterial-phase hyperenhancement and HCP hypointensity may be indistinguishable from small HCCs.

2. Acquired FNH-like lesions

Overview: These lesions are described in cirrhotic livers, and are macroscopically, microscopically and immunohistochemically identical to classic FNH seen in noncirrhotic livers. They are believed to originate from acquired hyperplastic responses to vascular alterations associated with cirrhosis, as opposed to conventional FNHs, which are believed to originate from developmental responses to congenital arterio-vascular malformations.

Unenhanced MRI features: Hypointense to isointense on T1-weighted MR images and mildly hyperintense to isointense on T2-weighted images. The "central scar", if present, is hyperintense on T2-weighted images.

Dynamic imaging with extracellular contrast agent: These lesions usually show intense enhancement in the arterial phase and fade to isointensity in the portal venous and later phases. Rarely, they may reveal a slight washout in the PV phase; marked washout is incompatible with FNH. [12].

Imaging with Gd-EOB-DTPA: Usually it enhances in the hepatobiliary phase (Fig. 4), but there have been reports of a few cases revealing hepatobiliary hypointensity [13].
Differential diagnosis: The major differential diagnosis is HCC, especially for small lesions with less than $3 \mathrm{~cm}$, those without a central scar and those with HCP hypointensity.

Potential pitfalls: While HCP hyperintensity is characteristic of FNH-like lesions, this finding is nonspecific and may also be observed in HCCs (see below). Favouring the diagnosis of a FNH-like lesion is the presence of a central scar and/or a network of fibrous septa carving the nodule into smaller lobules. Favouring HCC are the mosaic architecture, presence of a tumour capsule, mildly hyperintense signal intensity of T2-weighted, restricted diffusion on DW images or a combination of these.

While most FNH-like lesions exhibit diffuse HCP hyperintensity, these lesions may have central zones of portal venous phase washout and HCP hypointensity, mimicking HCC. In equivocal cases, close follow-up or biopsy should be considered (depending on lesion size and other factors).

\section{Hepatocellular carcinoma}

Overview HCC is the most common primary malignant hepatic tumour. Underlying cirrhosis from viral hepatitis (B and C), alcoholism, non-alcoholic steatohepatitis (NASH) and toxin exposure are the predominant causal factors. Tumour markers, such as serum-fetoprotein or PIVKA-II, are usually elevated in patients with HCC $[14,15]$. 


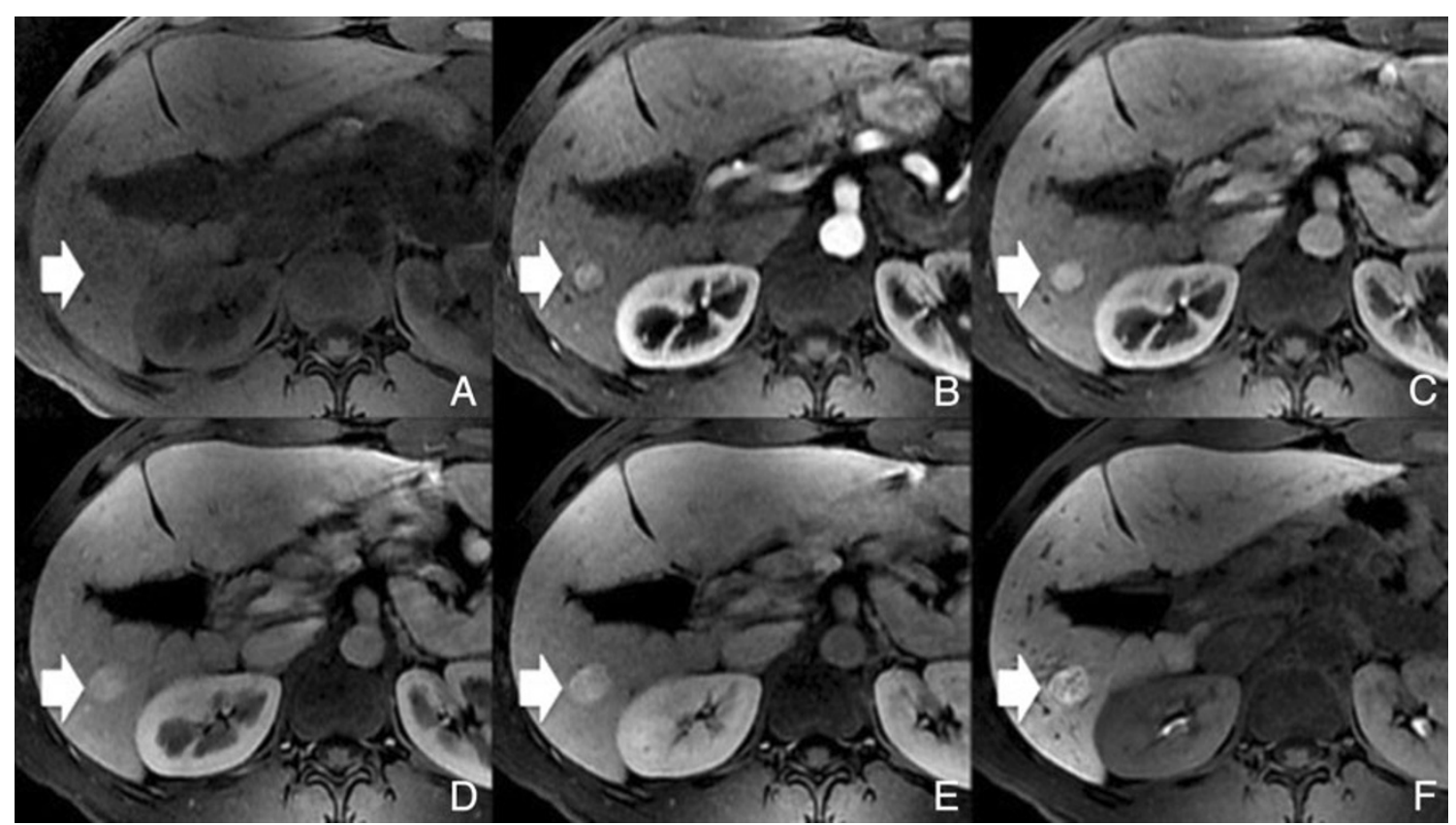

Fig. 4 An FNH-like lesion in a 41-year-old man with chronic liver disease. a Pre-contrast MRI, b arterial phase, c PV phase, d 5 min, e $10 \mathrm{~min}$, $\mathbf{f}$ hepatocellular phase. The image shows a liver with a lobulated contour. The arrows point to a small rounded focal lesion,

However, elevation of tumour markers does not help determine which of several lesions is malignant, if multiple nodules are observed, and negative tumour markers do not exclude HCC.

Unenhanced MRI features On T1-weighted MR images, $\mathrm{HCC}$ is most often hypointense relative to the liver, although hyperintense lesions or areas of hyperintensity within hypointense lesions may be seen [15]. On T2-weighted images, HCC is generally hyperintense, although some welldifferentiated HCC may be seen as isointense or mildly hypointense lesions. Steatotic HCCs are characterised by a signal loss on out-of-phase images relative to in-phase images (Fig. 5). Haemorrhagic hepatocellular carcinomas may have marked high signal intensity on T1-weighted images and low signal intensity on $\mathrm{T} 2$ - and $\mathrm{T} 2 *$-weighted images.

Dynamic imaging with extracellular contrast agent Typically, HCCs $(80 \%)$ show intense enhancement in the arterial phase contrast-enhanced images, with washout in the portal venous phase (Fig. 6). Less than $20 \%$ of the HCCs are hypovascular (usually well-differentiated tumours) [15, 16]. Small HCCs (most commonly well differentiated HCCs) are usually homogeneous. Large HCCs (most commonly moderately or poorly differentiated) may exhibit a broad spectrum of morphologic features, including a mosaic pattern (Fig. 7), a tumour capsule, an intratumoural nodule ("nodule-in-nodule" appearance), and an extracapsular isointense in the Pre-contrast sequence, showing brisk arterial enhancement. In the HCP it accumulates Gd-EOB-DTPA, exposing the typical FNH internal architecture

extension with the formation of satellite nodules. A tumour capsule shows higher enhancement than that of the surrounding parenchyma on delayed phase images. Capsular disruption, with extracapsular extension or absence of a capsule, is associated with poorer prognosis.

Imaging with Gd-EOB-DTPA In the hepatocellular phase, HCCs usually show low signal intensity. The degree and homogeneity of hypointensity is variable, depending on the concentration and function of cellular membrane receptors and transporters (OATPs and multidrug resistance-associated proteins [MRPs]). In some (2.5-8.5\%), uptake of the contrast agent can paradoxically occur (Fig. 8). Most of these hyperintense HCCs are well or moderately differentiated, with bileproducing or bile-containing capacity maintained [17-20].

Differential diagnosis Cholangiocarcinoma, arterioportal (AP) shunt, atypical haemangioma and hepatic adenoma are possible diagnosis. The usual occurrence in a cirrhotic liver, in addition to the dynamic and hepatocelullar phase imaging, usually permit the differential diagnosis.

Potential pitfalls Some HCCs will uptake Gd-EOB-DTPA in the hepatobiliary phase. These can usually be differentiated from RN, DN and FNH-like lesions because of their heterogeneity or mosaic pattern, the nodule-in-nodule appearance, the presence of a hypoenhancing rim (capsule) or the absence of a scar. 


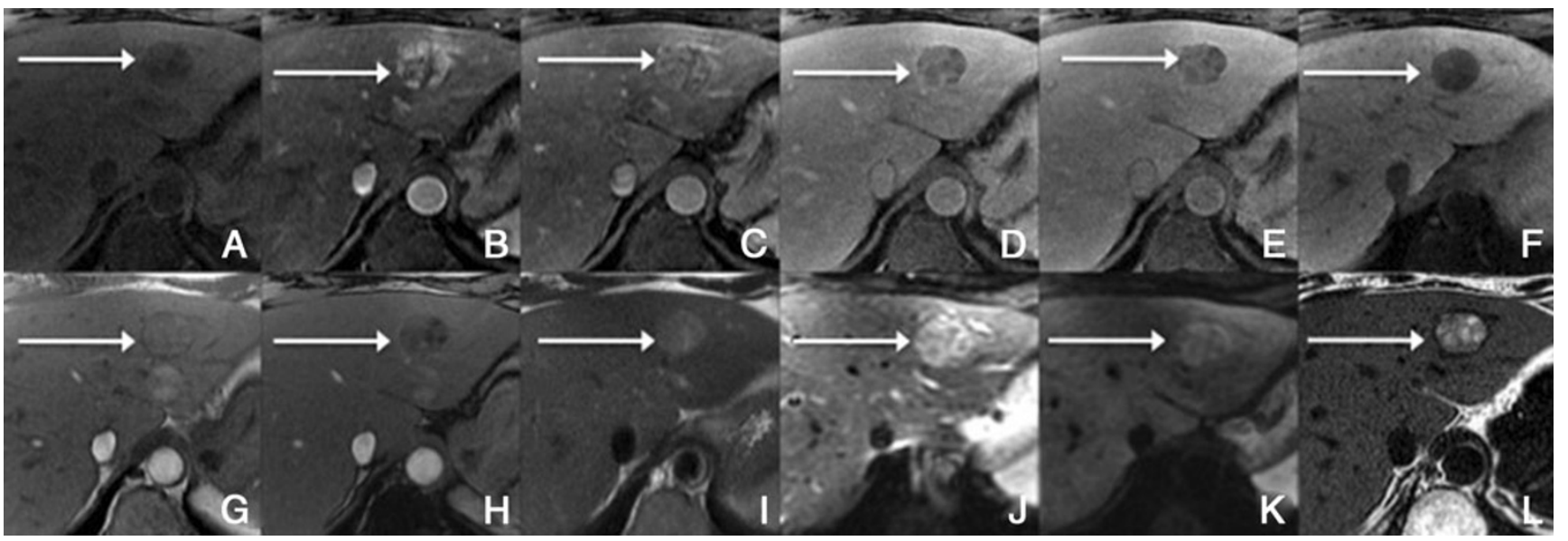

Fig. 5 Fatty HCC in a 45-year-old man with chronic liver disease. a Pre-contrast MRI, b, c arterial phase, d $3 \mathrm{~min}, \mathbf{f} 4 \mathrm{~min}, \mathbf{f} 8 \mathrm{~min}, \mathbf{g}$ IP, $\mathbf{h}$ OP, i SSFSE, $\mathbf{j}$ b0, $\mathbf{k}$ b500, 1 T1-weighted PDFF. The arrows point to a rounded focal lesion, with arterial enhancement, PV washout and

Infiltrative HCC may manifest as subtle heterogeneity and mild ill-defined hypointensity in the HCP. This may mimic background cirrhotic parenchyma and escape detection.

\section{Fibrolamellar HCC}

Overview Fibrolamellar HCC is an uncommon type of HCC affecting young adults.

Unenhanced MRI features It is usually seen as a large, wellcircumscribed focal lesion with low signal intensity on T1- hypointensity in the HCP. It also shows heterogeneous loss of signal in OP (fat), expressed likewise in the fat fraction map as bright areas in the lesion

weighted MR images and high signal intensity on T2-weighted images. A central radiating scar is seen in $80 \%$ of cases and has low signal intensity on T1- and T2-weighted images. Areas of liquefactive necrosis may occur centrally and appear markedly hyperintense on T2-weighted images.

Dynamic imaging with extracellular contrast agent There is usually early heterogeneous contrast enhancement, which fades on subsequent images. The scar has minimal or no enhancement on contrast-enhanced images, being usually hypointense relative to the remainder of the tumour (Fig. 9).

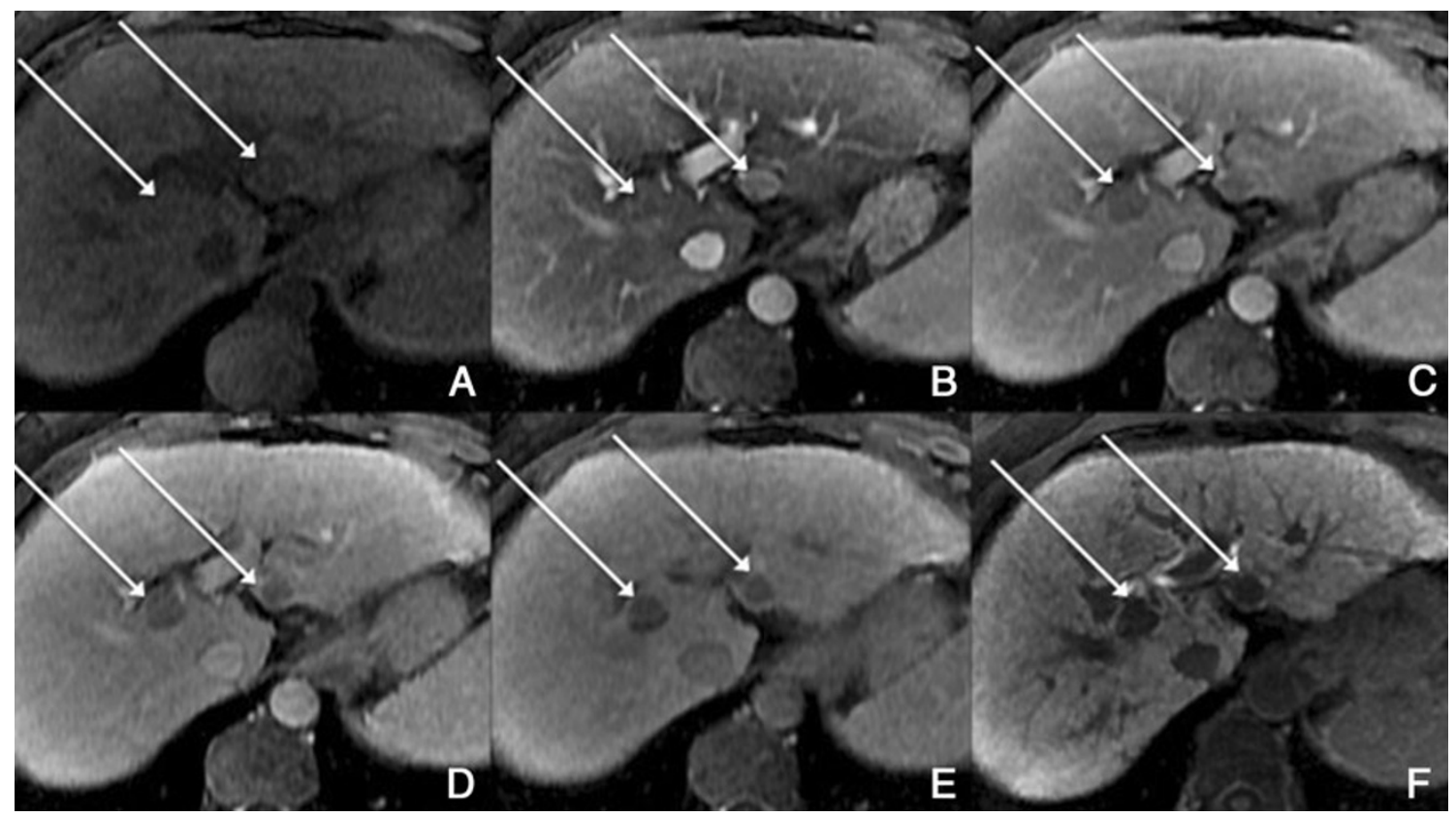

Fig. 6 Hypervascular HCCs in a 50-year-old man with chronic liver disease. a Pre-contrast MRI, b arterial phase, c portal vein pressure (PVP), d $3 \mathrm{~min}$, f $4 \mathrm{~min}$, f HCP. Marked heterogeneous liver with nodular contour, with reticulation in the HCP- cirrhotic The arrows point to two rounded focal lesions, with arterial enhancement (one less prominent), PV washout and hypointensity in the HCP 


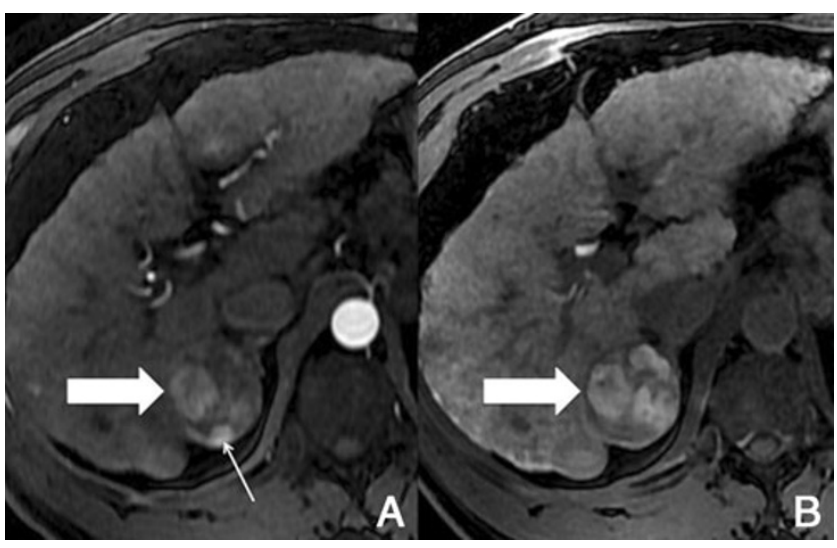

Fig. 7 A 52-year-old man with chronic liver disease. a Arterial phase, b HCP. Imaging findings of a cirrhotic liver, with a lesion (large arrows) with areas of enhancement (thin arrow) and non-enhancing areas - mosaic pattern. In the HCP we found a similar appearance with areas taking up Gd-EOB-DTPA and others not

Imaging with $G d-E O B-D T P A$ In hepatocellular phase some viable areas of the tumour may show some uptake of GdEOB-DTPA. The scar remains hypointense [21].

Differential diagnosis Hypointensity of the scar on T2weighted images and the hypointensity in the HCP distinguish fibrolamellar HCC from a FNH. It can be distinguished from HCC due to the presence of a scar, calcifications, larger size and occurrence in younger patients with no cirrhosis.
Potential pitfalls The absence of a scar and the uptake of Gd-EOB-DTPA in the HCP may be confounders.

Nodular perfusional alterations versus wedge-shaped perfusional alterations (transient hepatic intensity differences [THIDs])

Overview These perfusional alterations are phenomenon characterised by a patchy wedge-shaped area of enhancement involving a hepatic subsegment or by an arterial enhancing focal lesion that fades in the venous phase.

Unenhanced MRI features Generally, there are no alterations in morphology or signal intensity.

Dynamic imaging with extracellular contrast agent This finding is seen immediately after contrast material administration, with fading on subsequent images. They are thought to represent an imbalance between the hepatic arterial and portal venous supply, caused by increased hepatic arterial blood flow in the presence of portal vein obstruction (Figs. 10 and 11). Typically, transiently increased segmental perfusion enhancement indicates that the portal venous supply is compromised due to compression or thrombosis.

Imaging with Gd-EOB-DTPA No alterations in the hepatobiliary phase, due to intact hepatocyte function of these lesions, make it possible to differentiate from small HCCs [22, 23].

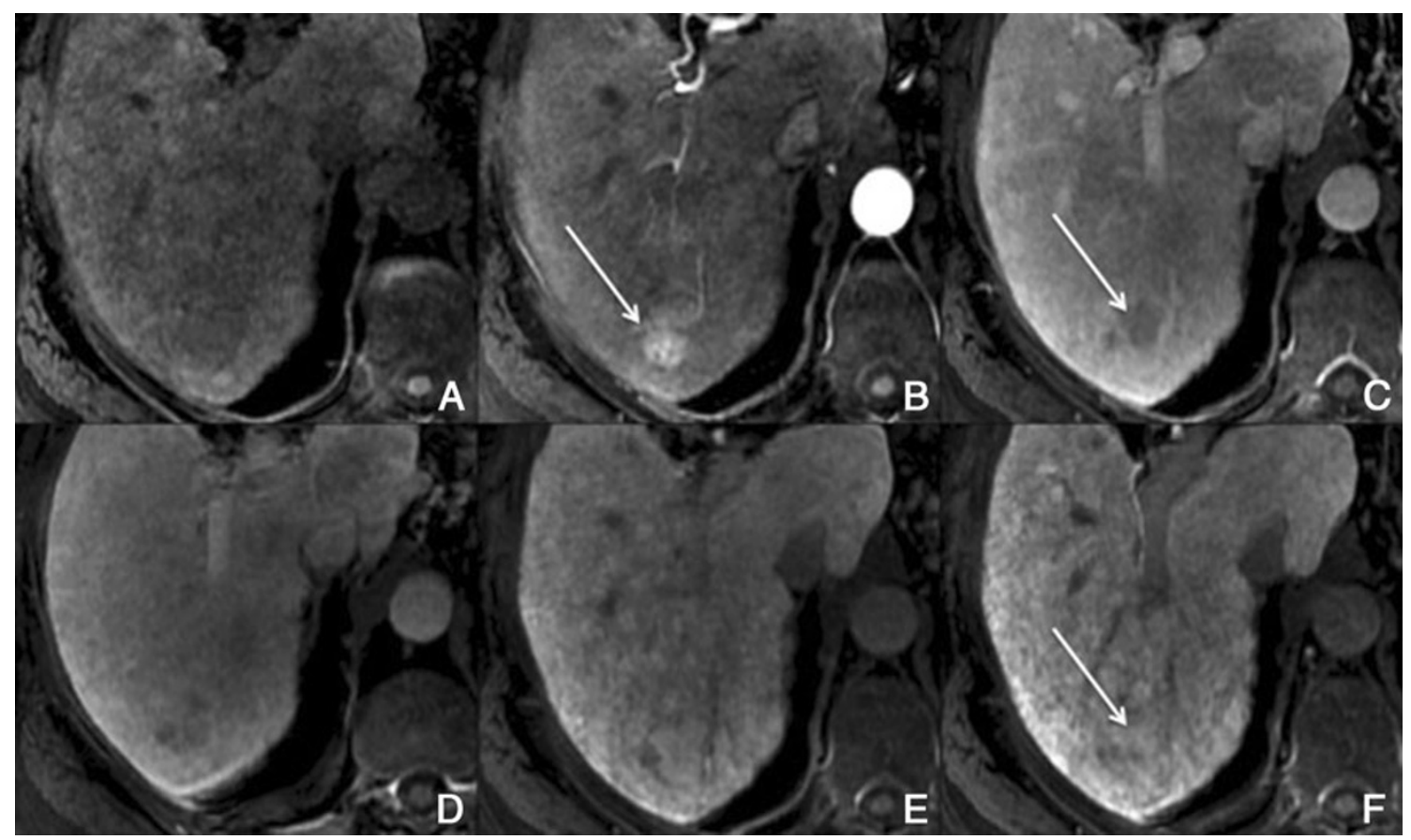

Fig. $8 \mathrm{HCC}$ in a 42-year-old woman with chronic liver disease. a Pre-contrast MRI, b arterial phase, c PV phase, d 5 min, e 10 min, f HCP. Hypervascular HCC, with washout in the PV phase and uptake of Gd-EOB-DTPA (arrows) 
Fig. 9 Fibrolamellar-HCC in a 34-year-old man without liver disease. a Pre-contrast MRI, b arterial phase, $\mathbf{c}$ PV phase, $\mathbf{d}$ $\mathrm{HCP}$. Big nodular lesion in the left lobe isointense in precontrast imaging, with arterial enhancement, washout in the PV phase and no uptake of GdEOB-DTPA in the HCP (thick arrow). The central scar (thin arrow) remains hypointense in all sequences

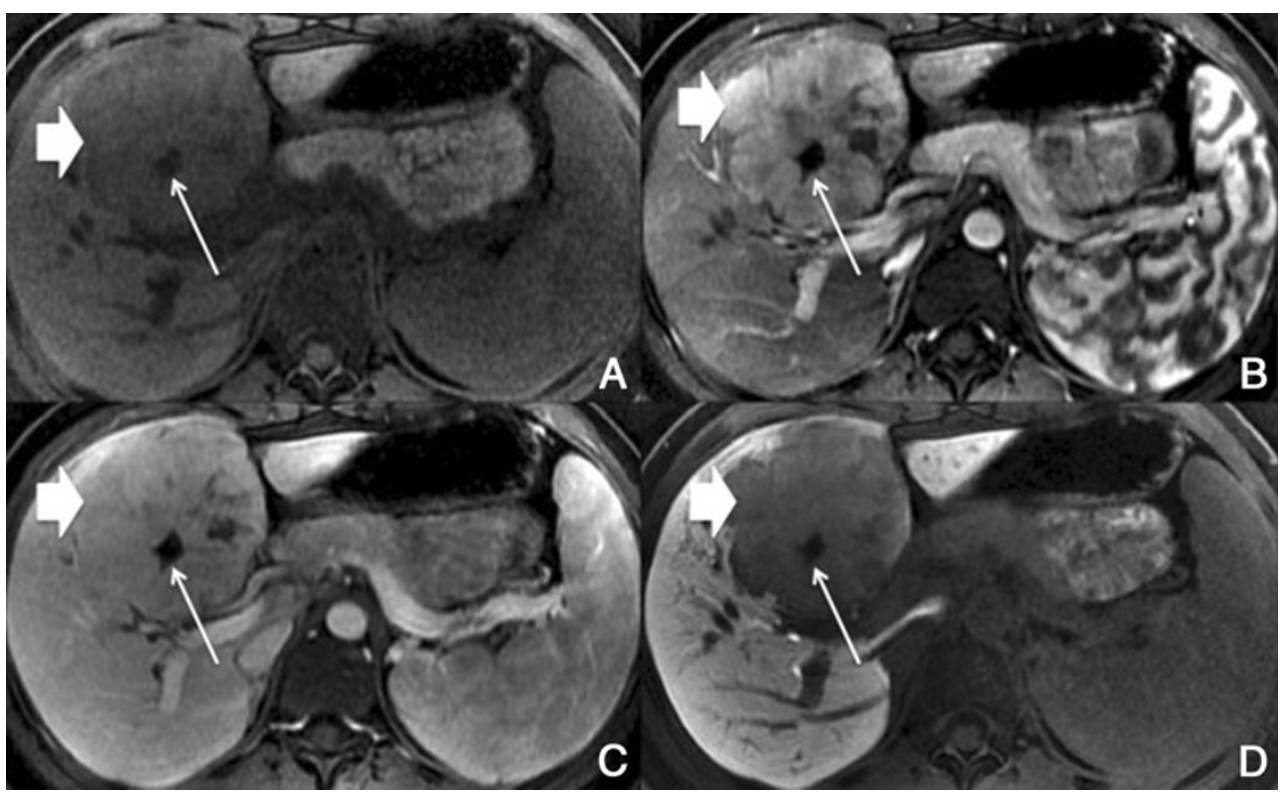

Differential diagnosis Small HCCs and hypervascular metastases are possible diagnosis. Usually the absence of a defect in the HCP makes the diagnosis of THID.

Potential pitfalls Rarely, vascular alterations are associated with dysfunction of the hepatocytes, which reveal a defect in the HCP, complicating the diagnosis.

\section{Focal nodular hyperplasia (FNH)}

Overview FNH is the second most common benign tumour of the liver, after hepatic haemangiomas. FNH typically occurs as a solitary lesion in young female patients. It consists of aggregates of hepatocytes and is thought to be secondary to a proliferative response of hepatocytes, secondary to an underlying vascular malformation. Biliary structures proliferate without connection to the adjacent biliary tree. Kupffer cells are present, often in greater amounts than in the surrounding normal liver parenchyma. The central scar in FNH is not a true scar, but represents a congeries of blood vessels, bile ducts and sometimes a focal area of cirrhosis. About $20 \%$ of FNH cases are classified as non-classic. These non-classic FNH subtypes lack the findings of nodular architecture or malformed vessels but always have bile duct proliferation.

Unenhanced MRI features The lesion varies from mildly hypointense to isointense on T1-weighted MR images and from mildly hyperintense to isointense on T2-weighted images. The "central scar" is characteristically hyperintense on T2-weighted images. The hyperintensity of the lesion on T2-weighted images may be related to the presence of vascular channels or oedema throughout the lesion.

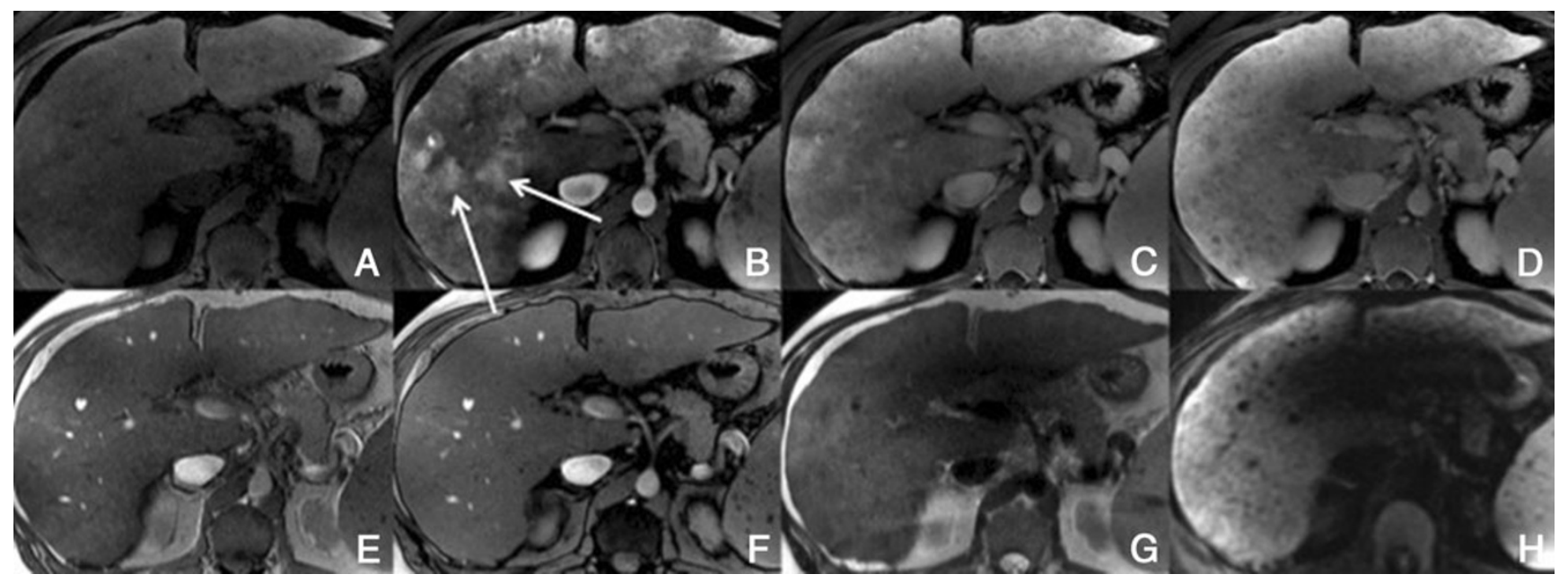

Fig. 10 THIDs in a 38-year-old man with chronic liver disease. a Pre-contrast MRI, b arterial phase, c 2 min, d 3 min, e IP, f OP, g T2, h DWI B500. Heterogeneous liver with nodular contour. Fluffy hyperintense areas in the arterial phase (arrows), not seen in the other sequences 
Fig. 11 Multiple THIDs and a big HCC in a 47-year-old man with chronic liver disease. a Pre-contrast MRI, b arterial phase, $\mathbf{c}$ PV phase, $\mathbf{d}$ delayed phase. Big nodular lesion in the left lobe hypointense in precontrast imaging, with arterial enhancement, internal arteries, washout in the PV phase and heterogeneous uptake of GdEOB-DTPA in the HCP $\left(^{*}\right)$. There also seen multiple nodulariform hyperintense areas in the arterial phase (arrows), not seen in the other sequencesTHIDs

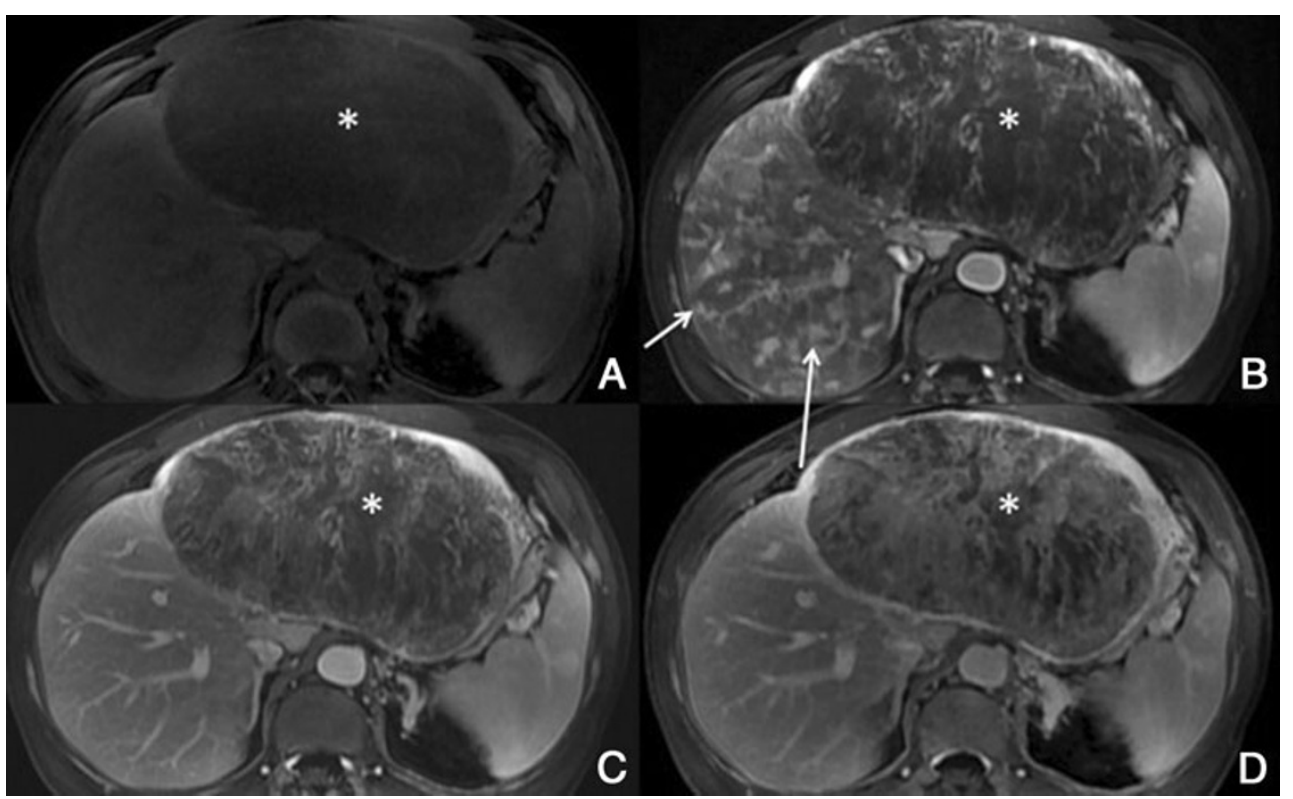

Dynamic imaging with extracellular contrast agent $\mathrm{FNH}$ is perfused by the hepatic arterial system and shows marked, nearly uniform arterial-phase enhancement. The degree of lesion enhancement lessens on subsequent contrast-enhanced images, with lesion signal intensity approaching that of the surrounding liver parenchyma. The central scar has low signal intensity on early phase contrast-enhanced images but gradually enhances to become hyperintense relative to the rest of the lesion on delayed phase images [24, 25]. The capsule is usually absent.

Imaging with Gd-EOB-DTPA With hepatobiliary-specific agents, it shows homogenous or heterogeneous enhancement in the hepatocellular phase, as it lacks a well-formed bile canalicular system for normal excretion. It becomes isointense to hyperintense to the normal liver, allowing the differential diagnosis $[8,26]$. More than the enhancement itself, the hepatocellular phase also permits the evaluation of the internal architecture (reticulation or lace-like appearance), which provides more certainty in the diagnosis (Fig. 12).

Differential diagnosis Adenoma and fibrolamellar HCC. The isointensity to hyperintensity in the HCP and the imaging features of the central scar of FNH are usually helpful in the differential diagnosis. The adenoma does not present a central scar and is hypointense in the HCP. The F-HCC is usually larger, more heterogeneous and its scar is hypointense in every sequence.

Also, the non-classic forms often present a diagnostic challenge in imaging: e.g. telangiectatic $\mathrm{FNH}$ has been shown to demonstrate atypical imaging features, including lack of a central scar, high signal intensity on T1- and T2weighted images and persistent enhancement on delayed phase images [26].

Potential pitfalls About $12 \%$ of FNH can show atypical imaging features on Gd-EOB-DTPA enhanced MRI, such as the absence of a scar or an eccentric one, or washout in the venous phase.

\section{Adenoma}

Overview Hepatic adenomas are rare benign tumours of the liver. On histopathological analysis, hepatic adenomas contain well-differentiated hepatocytes lacking bile ducts or portal tracts. The majority of adenomas are solitary (80\%) and typically occur in female patients (90\%). Predisposing factors to adenoma formation include oral contraceptive use in female patients, anabolic steroid use in male patients and glycogen storage disease [27].

Unenhanced MRI features They are generally well circumscribed, without contour lobulation and have variable degrees of haemorrhage, necrosis, fat and, rarely, calcification. At MR imaging, adenomas are typically hyperintense to isointense on T1-weighted images and slightly hyperintense on T2-weighted images.

Dynamic imaging with extracellular contrast agent After administration of contrast material, adenomas typically demonstrate moderate enhancement on arterial phase images; they may show washout and enhancement similar to that of surrounding liver parenchyma on portal venous and delayed phase images [28]. 


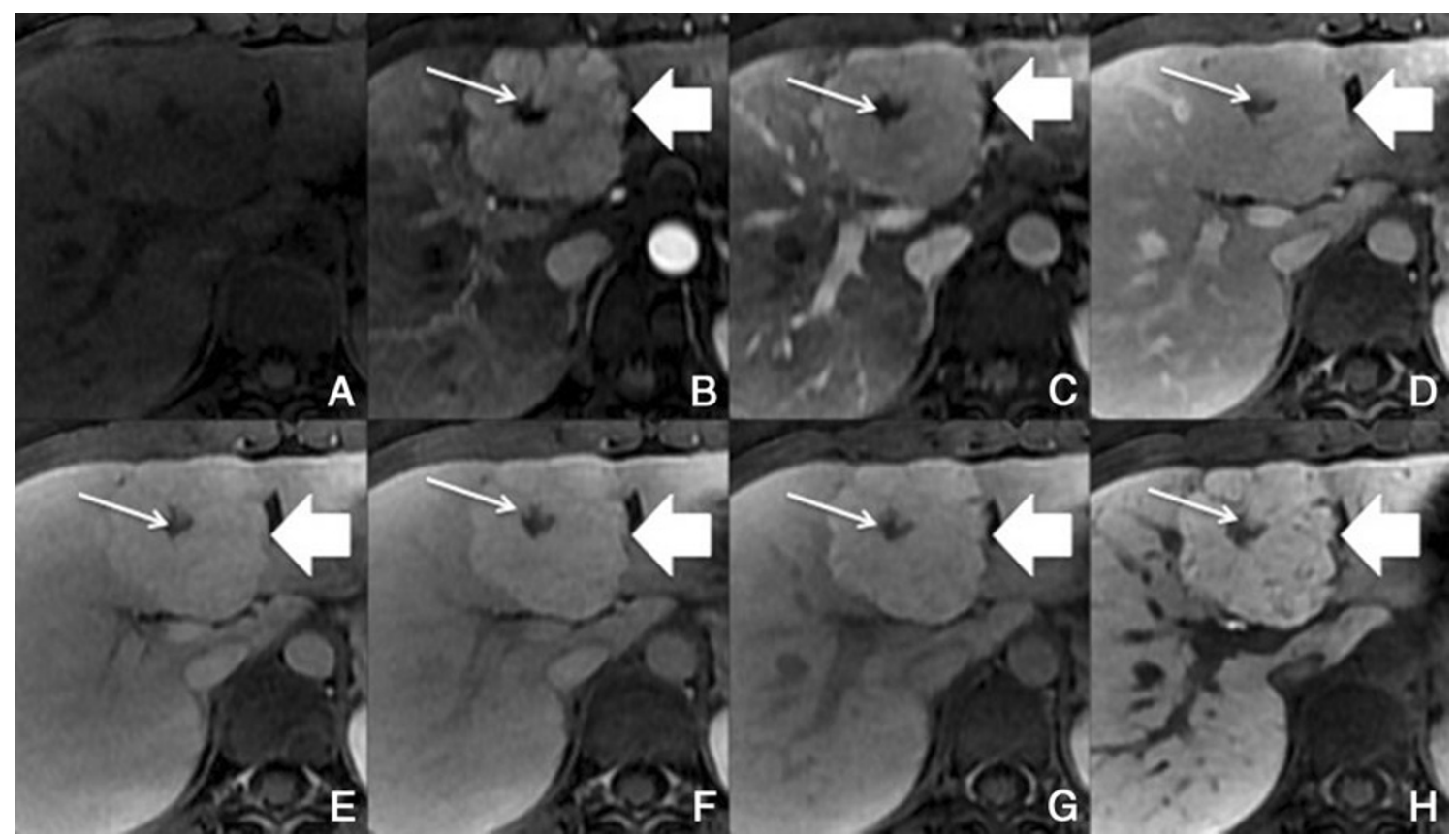

Fig. 12 FNH in a 37-year-old woman with no liver disease. a Precontrast MRI, b, c arterial phase, d $3 \mathrm{~min}$, e $5 \mathrm{~min}$, f $8 \mathrm{~min}, \mathbf{g ~} 10 \mathrm{~min}$, h HCP. Big nodular lesion (thick arrows), with lobulated contours isointense in pre-contrast imaging, with arterial enhancement, washout

Imaging with Gd-EOB-DTPA Usually no enhancement of hepatic adenoma is seen in the hepatocellular phase because of absent or strongly reduced hepatocellular uptake of Gd-EOB- in the PV phase and uptake of Gd-EOB-DTPA in the HCP - note the fine reticular architecture typical of a FNH. The central scar (thin arrow) remains hypointense in all sequences

DTPA (Fig. 13). However, peripheral or faint diffuse enhancement has been reported in some cases due to some hepatocytes maintaining the capability of uptake and excretion in the HCP.

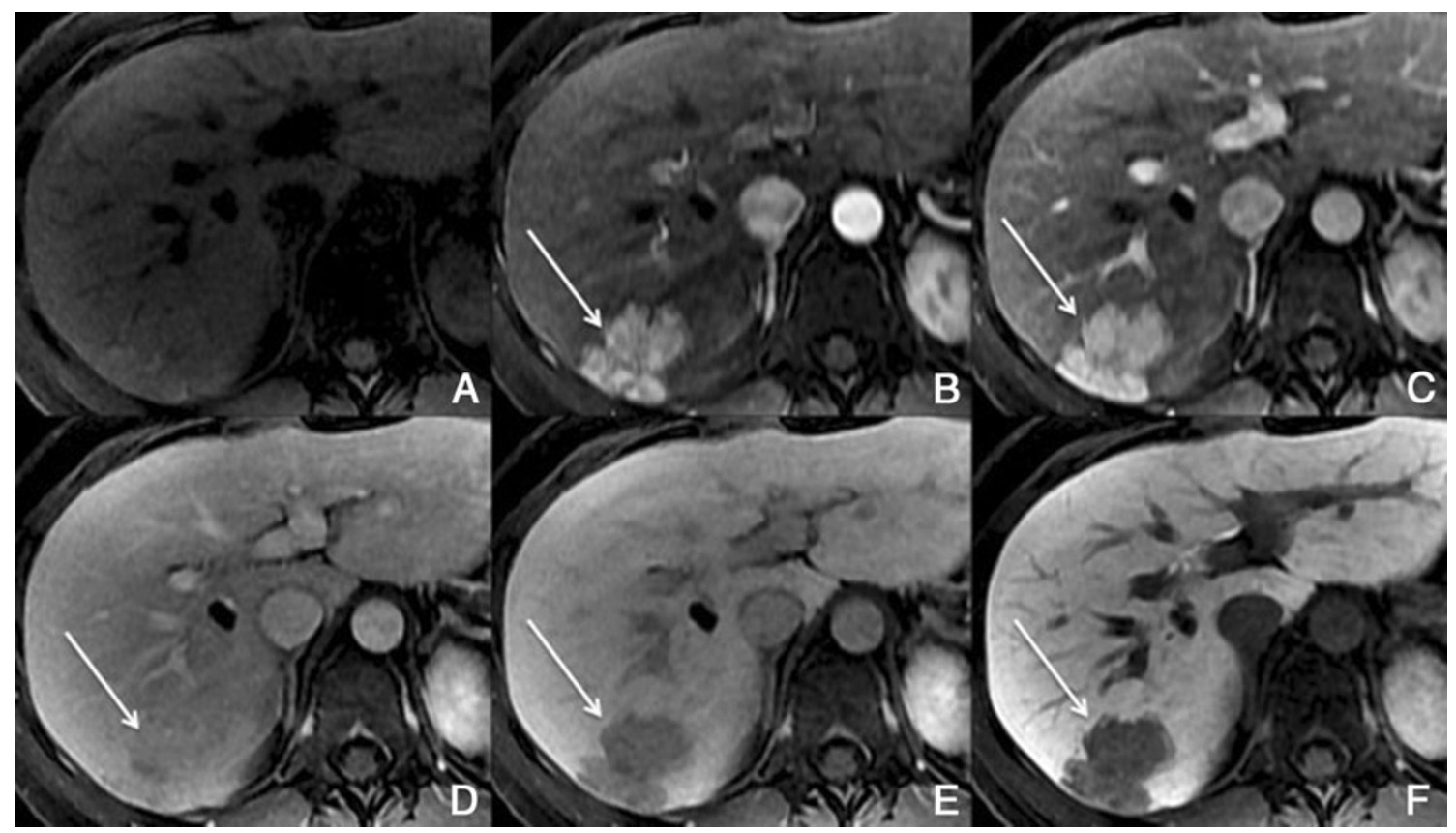

Fig. 13 Liver adenoma in a 35-year-old woman on oral contraceptives, with no liver disease. a Pre-contrast MRI, b, c arterial phase, $\mathbf{d}$ $3 \mathrm{~min}$, e $5 \mathrm{~min}$, f HCP. Lobulated lesion in the right lobe isointense in pre-contrast imaging, with arterial enhancement, internal arteries and no uptake of Gd-EOB-DTPA in the HCP (arrow) 


\section{Differential diagnosis $\mathrm{FNH}$ and $\mathrm{HCC}$.}

The age and gender of the patient, occurring in a noncirrhotic liver (as opposed to HCC), the absence of a scar or the enhancement pattern, usually helps in the diagnosis, since $\mathrm{FNH}$ is isointense to hyperintense in the $\mathrm{HCP}$, while the adenoma is typically hypointense.

Potential pitfalls The adenoma may show faint enhancement in the HCP, which is a confounder, but the other imaging characteristics usually permit the diagnosis.

\section{Focal hypereosinophilic infiltration (FEI)}

Overview FEI of the liver is a focal hepatic lesion caused by eosinophil-related tissue damage. It is associated with various eosinophilia-related conditions such as parasitic infections, allergic reactions, hypereosinophilic syndrome and tumours.

Unenhanced MRI features They have an indistinct margin and a non-spherical shape [29, 30]. On T1- and T2-weighted images, visible focal eosinophilic infiltrations and eosinophilic abscesses exhibit low and high signal intensities, respectively.

Dynamic imaging with extracellular contrast agent The dynamic imaging is characterised by poor enhancement on the arterial phase with homogeneous enhancement on portal venous phase images.

Imaging with $G d-E O B-D T P A$ They show mixed hypointensity, irregular margins and non-spherical shapes in hepatocellular phase (Fig. 14).
Differential diagnosis Metastases and abscesses. The nonspherical shape and irregular margins favour FEI.

Potential pitfalls The differential diagnosis from metastases in a patient with known primary malignancy is still challenging.

\section{Confluent fibrosis}

Overview Focal fibrosis occurs in the process of hepatic parenchyma collapse and its replacement with focal fibrotic masses, in the context of cirrhosis. It is usually located in the anterior and medial segments of the liver and has a wedgeshaped appearance. In some patients the entire segment is involved.

Unenhanced MRI features Fibrosis can appear as an area of low signal intensity on T1-weighted MR images and high signal intensity on T2-weighted images. It shows a typical geographic pattern of involvement, associated to retraction of the liver capsule.

Dynamic imaging with extracellular contrast agent It usually demonstrates delayed enhancement on contrastenhanced images.

Imaging with $G d-E O B-D T P A$ It shows low signal intensity due to decreased hepatic function from fibrosis (Fig. 15).

Differential diagnosis Infiltrative HCC and cholangiocarcinoma. Confluent fibrosis can be differentiated from infiltrative tumours on the basis of typical geographic appearance, location, no adjacent vascular invasion and no surrounding ductal dilatation.
Fig. 14 Focal

hypereosinophilic necrosis nodule in a 55-year-old man with no liver disease. a Arterial phase, b PV phase, c HCP, d T2-weighted. Small nonspherical lesion with ill-defined margins in the right lobe with poor enhancement in the arterial and PV phase, mixed hypointensity on the HCP and slightly hyperintense in $\mathrm{T} 2$-weighted (black arrows). This lesion was biopsy proven to be a lesion of focal eosinophilic infiltration

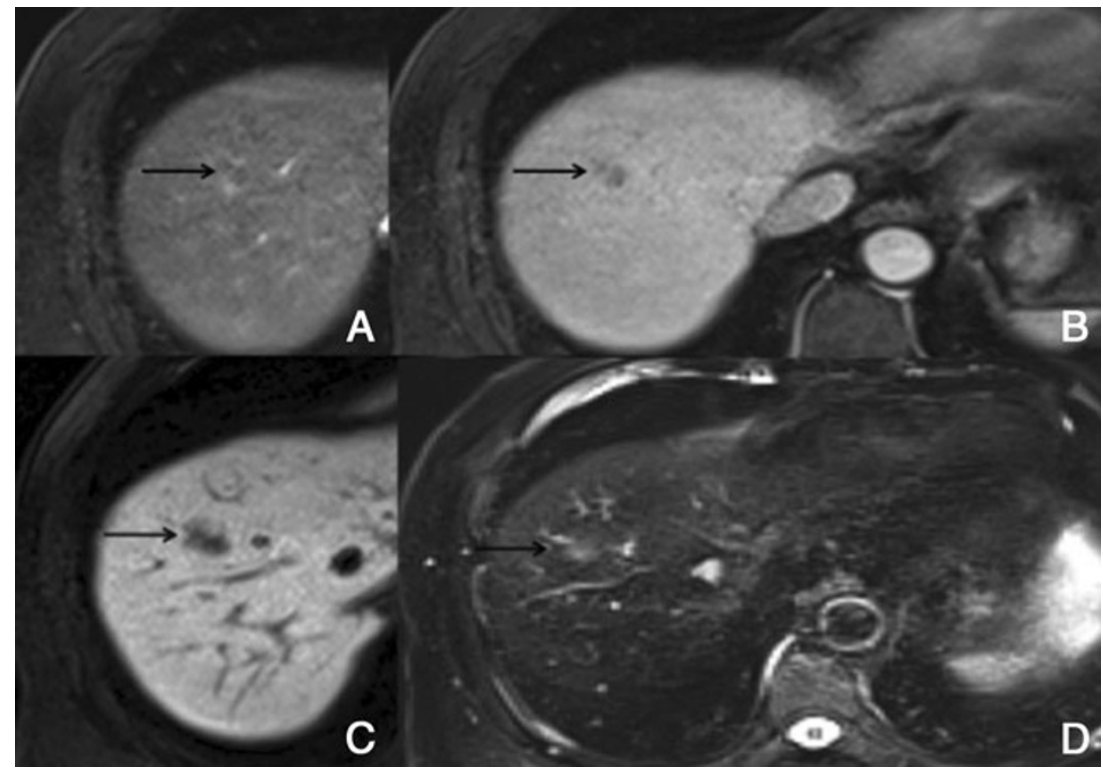




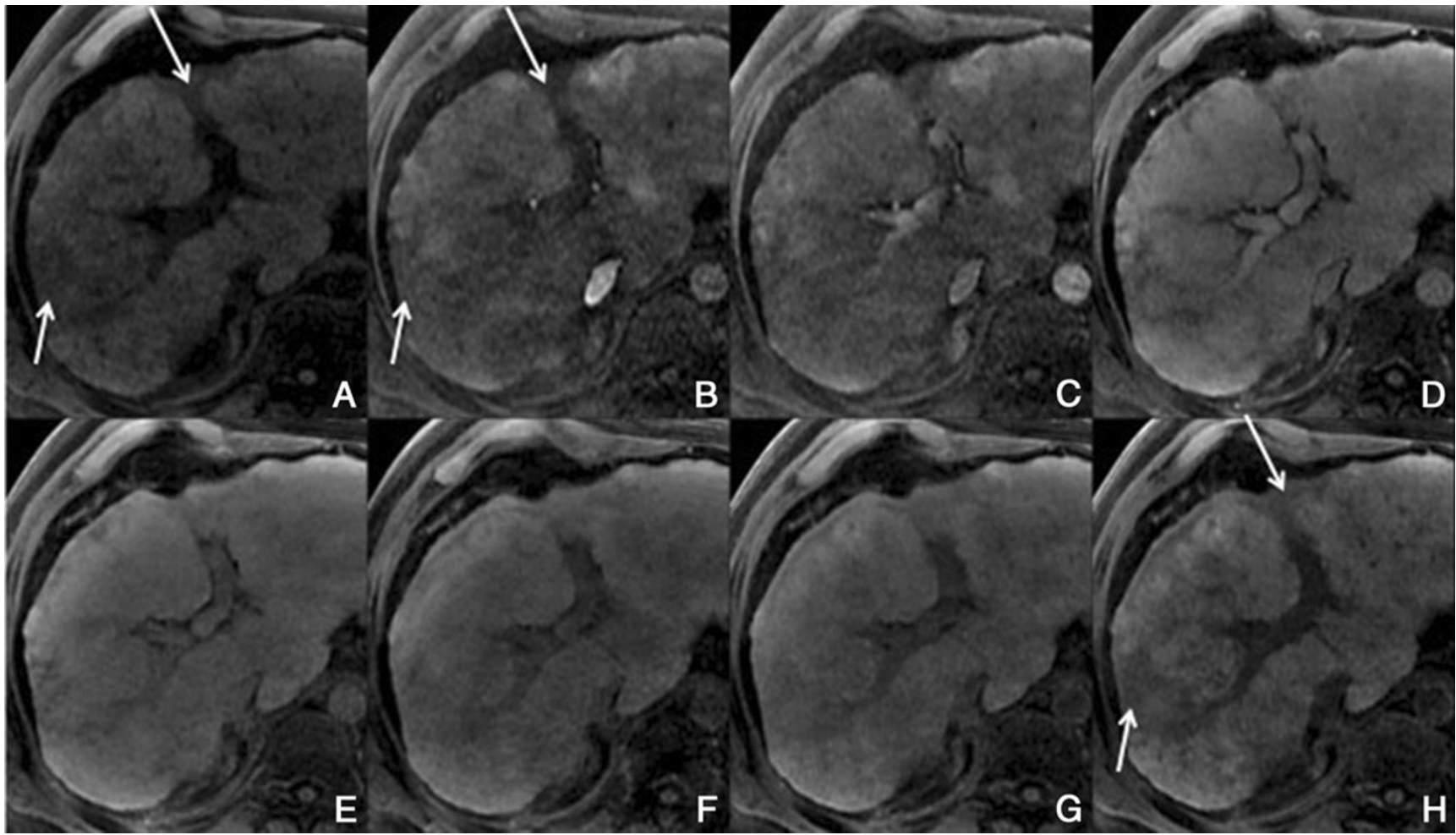

Fig. 15 Confluent fibrosis in a 56-year-old man with cirrhosis. a Precontrast, $\mathbf{b}$, $\mathbf{c}$ arterial phase, d PV phase, e $3 \mathrm{~min}$, f $5 \mathrm{~min}, \mathbf{g} 8 \mathrm{~min}$, h HCP. Wedge-shaped ill-defined areas associated with capsular retraction, with decreased enhancement in the dynamic phases and with no uptake of Gd-EOB-DTPA in the HCP (arrows)

Dynamic imaging with extracellular contrast agent On enhanced MRI, there is a typical peripheral nodular enhancement with centripetal progression, resulting in diffuse high signal intensity on delayed phase images. Less commonly, flash filling of an entire small haemangioma or enhancement of small portions of the lesion, on arterial phase images, may be seen. Larger lesions may not demonstrate uniform enhancement on delayed phase images, probably due to areas of thrombosis or insufficient delay time. Central scars may be seen in hepatic haemangiomas.

Imaging with Gd-EOB-DTPA With this contrast, the enhancement of a haemangioma tends to follow the blood vessels. Hepatic haemangiomas will "pseudo washout" to become darker than the liver because of rapid uptake of the contrast agent by the surrounding normal liver during equilibrium phase (Fig. 16). However, careful analysis usually permits differential diagnosis from malignant lesions that washout, as malignant lesions will washout more rapidly than the vessels, while haemangiomas will parallel the vessels [31].

Differential diagnosis Small haemangioma may mimic hypervascular metastasis. In these cases, the bright signal of haemangiomas in heavily $\mathrm{T} 2$-weighted sequences is 


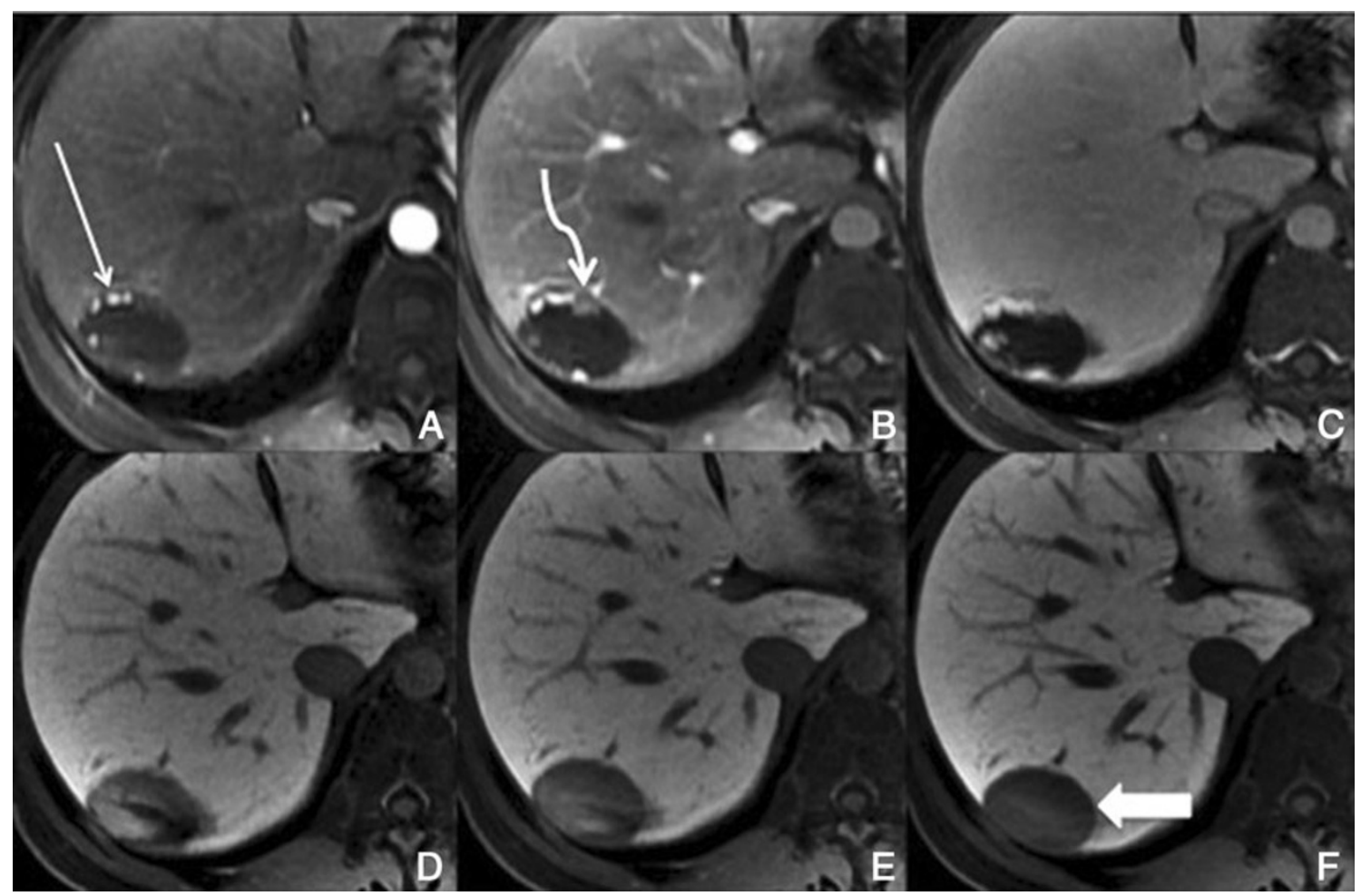

Fig. 16 Haemangioma in a 39-year-old woman with no liver disease. a, b Arterial phase, c PV phase, d $3 \mathrm{~min}$, e $5 \mathrm{~min}$, f HCP. Right lobe hypointense lesion with arterial nodular peripherical enhancement (thin arrow) with centripetal progression. Note that the hepatocellular uptake starts immediately (curved arrow). The intralesional enhancement follows the blood pool. In the HCP the lesion is hypointense because of rapid uptake of Gd-EOB-DTPA by the surrounding normal liver during equilibrium phase (thick arrow)
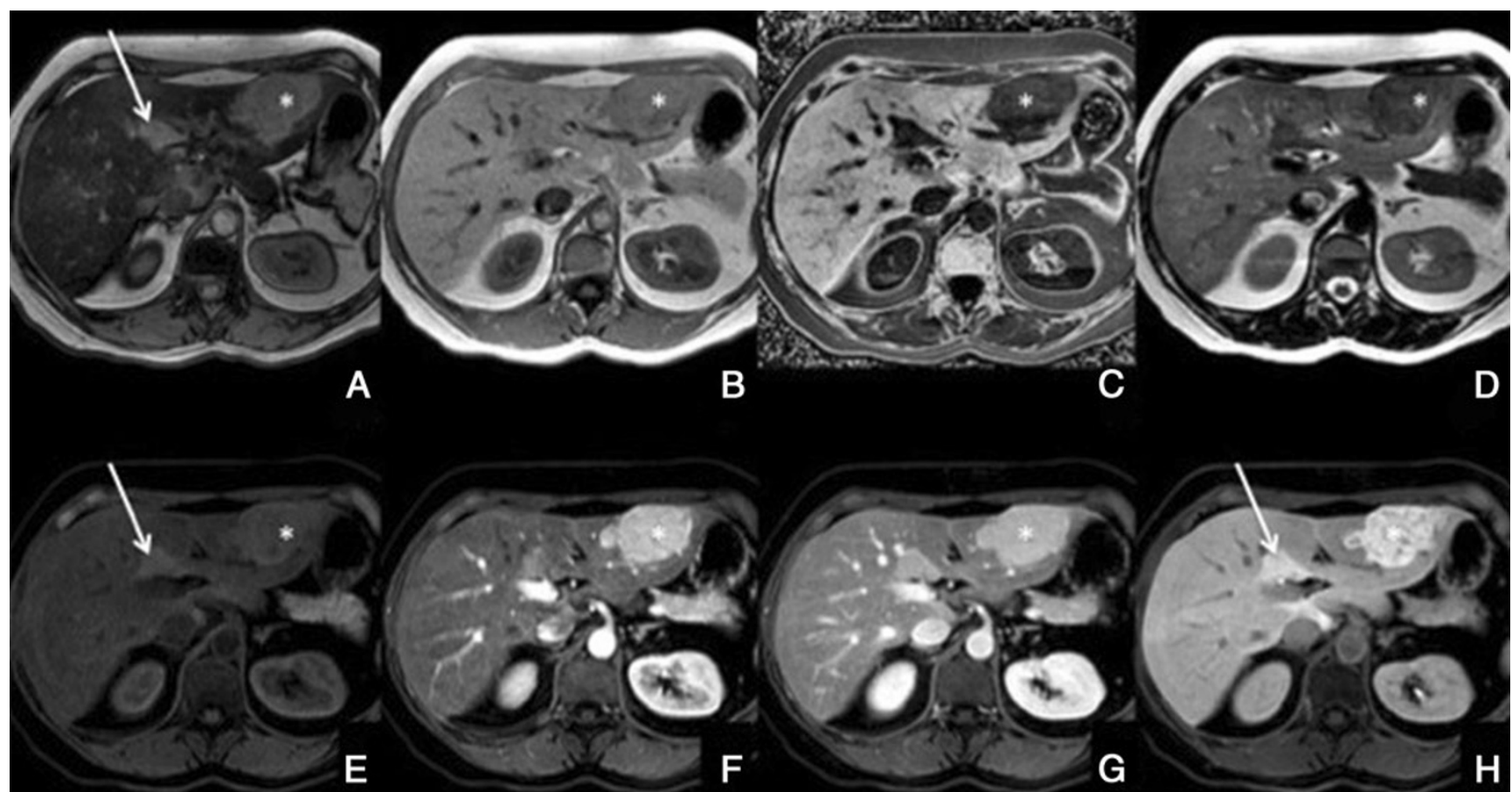

Fig. 17 Focal fat sparing area and a FNH, in a 36-year-old woman with no liver disease. a OP, b IP, c PDFF, d T2-weighted, e T1 fat-sat, f arterial phase, g PV phase, h HCP. Triangular-shaped periportal area (arrows) hyperintense to the rest of the liver in OP and Fat Sat imaging mimicking uptake of Gd-EOB-DTPA, corresponding to a fat spared area. This is confirmed in the fat-fraction map. Of note, is a lesion $\left(^{*}\right)$ compatible with a FNH 


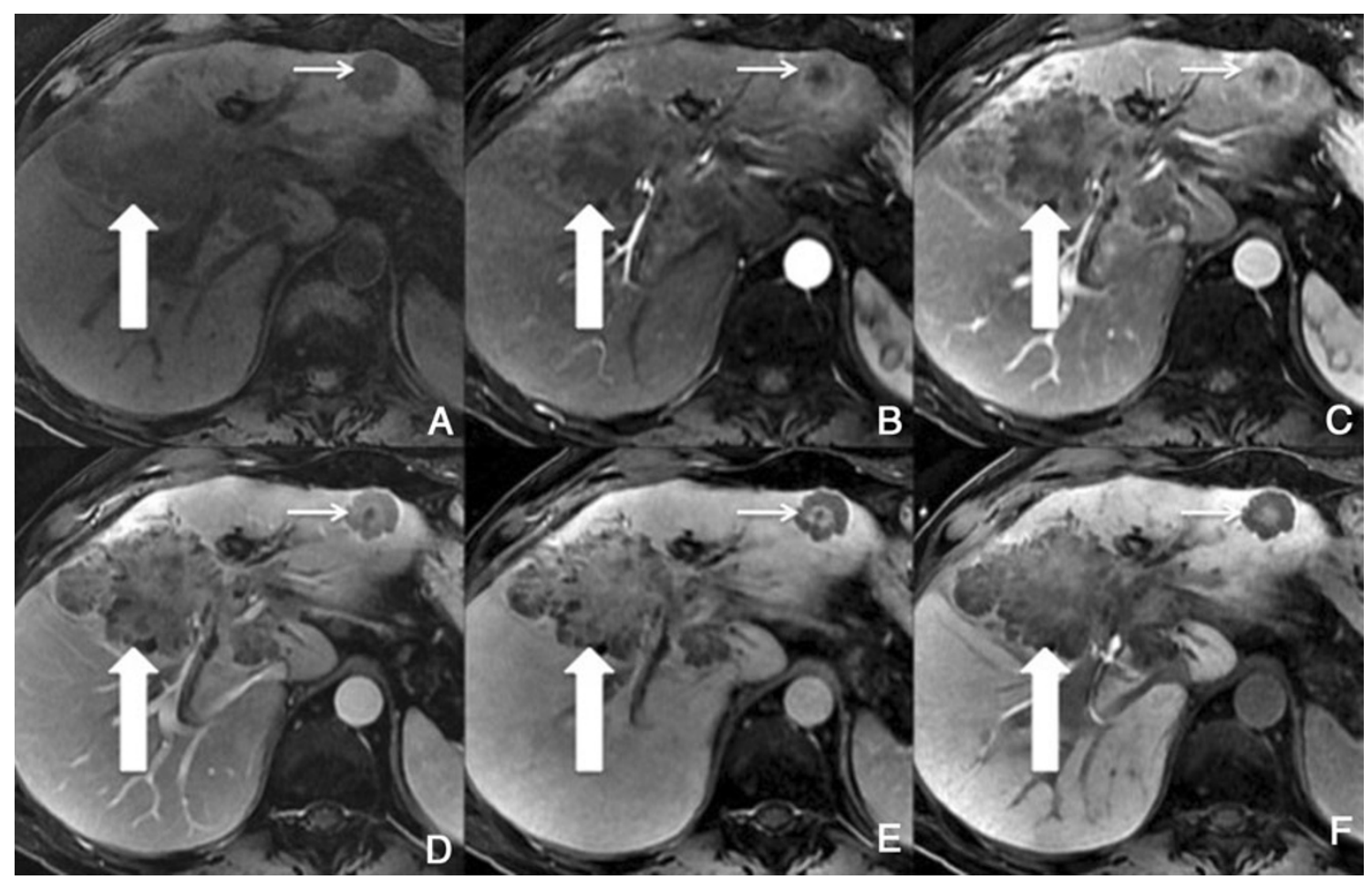

Fig. 18 Cholangiocarcinoma with a satellite lesion, in a 62-year-old man. a Pre-contrast, b, c arterial, d $3 \mathrm{~min}$, e $5 \mathrm{~min}$, f HCP. Large massforming lesion, irregular contour, with mild heterogeneous enhancement in the dynamic phases, with no uptake of Gd-EOB-DTPA in the
HCP (thick arrow) There is another lesion (thin arrow) in the left lobe with similar contrast uptake proprieties and a necrotic centre, compatible with a metastasis

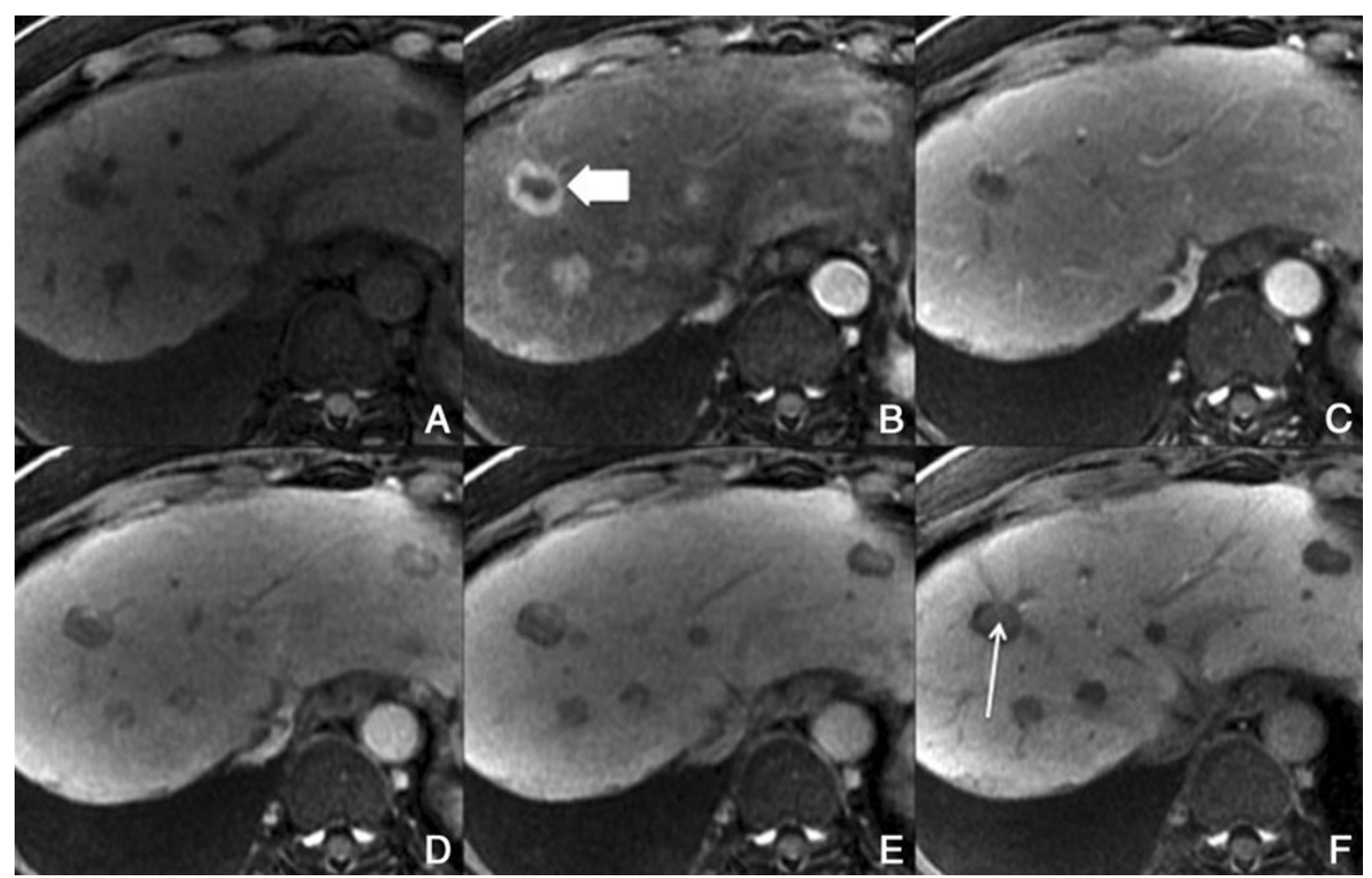

Fig. 19 Multiple hypervascular metastases, in a 39-year-old woman with breast cancer. a Pre-contrast, b arterial phase, c PVP, d 3 min, e $5 \mathrm{~min}, \mathbf{f ~ H C P}$. Multiple nodular lesions are seen, hyperenhancing in the arterial phase, with washout in the PVP, some with a hypointense necrotic centre (solid arrow). In the HCP, some of these lesions paradoxically uptake Gd-EOB-DTPA in the necrotic centre (thin arrow) 
helpful. Furthermore, in the dynamic phases, the intralesional degree of enhancement is similar to that of the adjacent vessels, pointing to a lesion filling with less enhanced blood due to the uptake of the hepatocytes.

Potential pitfalls In patients with prolonged intravascular dwell time due to hepatic or renal insufficiency, haemangiomas may appear isointense to the liver in the hepatocellular phase rather than hypointense. The key is parallelism to the blood pool.

\section{Focal fat/focal sparing}

Overview Focal fatty deposition and focal fatty sparing may manifest as a "pseudolesion". Clues to this diagnosis are its more common occurrence in the following areas of the liver: the medial aspect of the left lobe, along the falciform ligament, round the gallbladder fossa and within the central aspect of segment IV, adjacent to the porta hepatis. In addition, focal fatty infiltration is not seen to exert mass effect and vessels are seen to pass through these areas unimpeded.
Unenhanced MRI features MR imaging provides a useful method for characterising suspected geographic fatty infiltration by using in-phase and opposed-phase gradient-echo T1-weighted sequences. The relative increase in intravoxel fat in areas of focal fatty infiltration demonstrates decreased signal intensity on the opposed-phase images in comparison to the in-phase images. On MR imaging, areas of fatty sparing may be identified due to the lack of decrease in signal intensity on opposed-phase gradient-echo T1-weighted images and appear hyperintense relative to surrounding steatosis. Focal fat often appears dark on opposed-phase images, while focal sparing often appears bright [32].

Dynamic imaging with extracellular contrast agent Focal fat deposition and fat sparing areas show similar degree of enhancement to surrounding hepatic parenchyma.

Imaging with $G d-E O B-D T P A$ Areas of focal fat sparing area can be shown as mildly increased signal intensity on the hepatobiliary phase because the signal intensity of the focal fat sparing area is already higher on the unenhanced fat saturated sequence (Fig. 17).

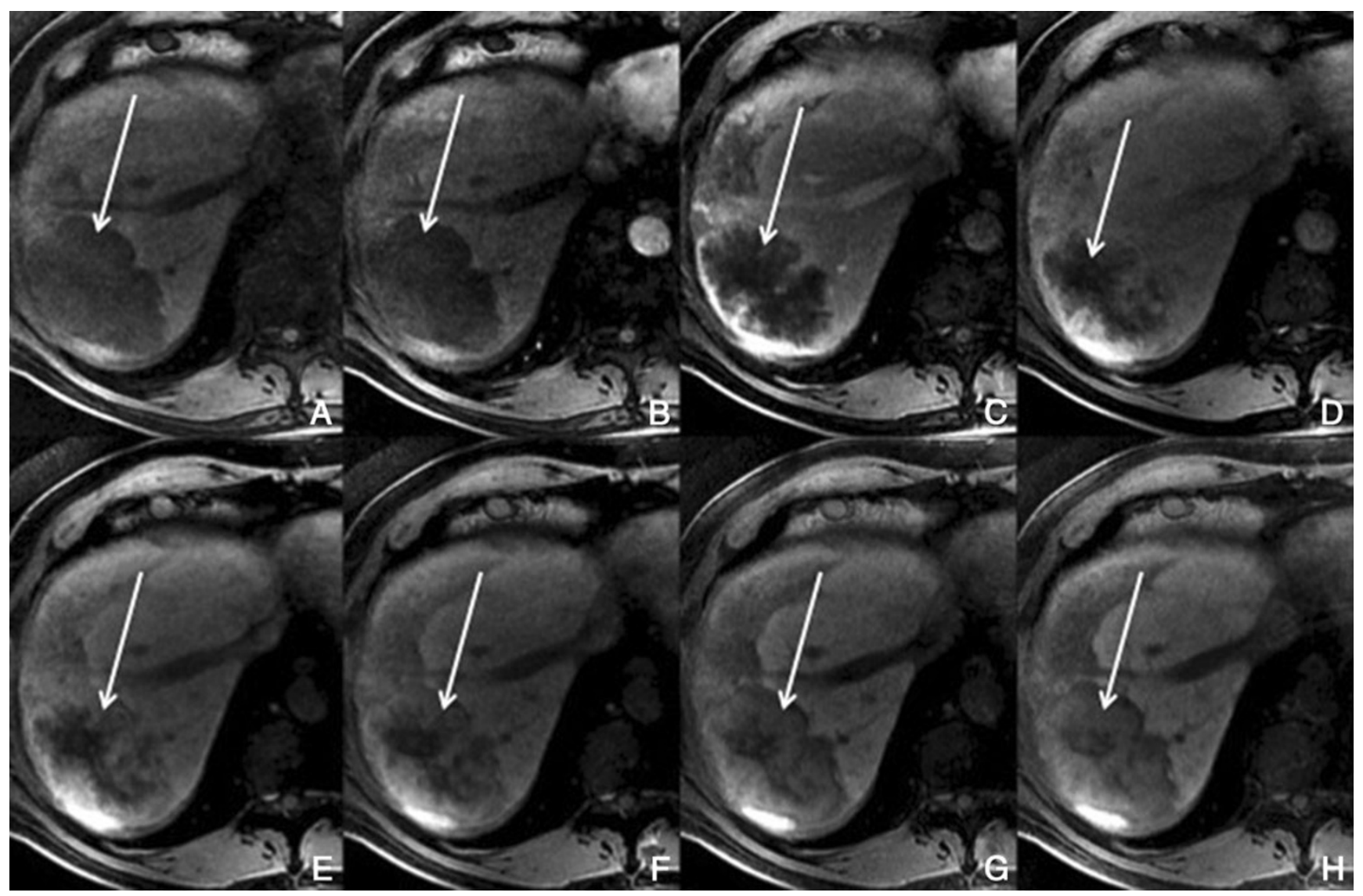

Fig. 20 Multiple hypovascular metastases, in a 60-year-old man with an NE tumour. a Pre-contrast, b, c arterial, d $3 \mathrm{~min}$, e $5 \mathrm{~min}, \mathbf{f} 8 \mathrm{~min}, \mathbf{g}$ $10 \mathrm{~min}, \mathbf{h}$ HCP. Multiple hypointense nodular lesions, with no significant enhancement in the dynamic phases, one of large dimensions in the right lobe (arrows), with progressive central pooling of GdEOB-DTPA, more evident in the HCP 
Differential diagnosis Focal hepatic lesion and lipoma. The typical locations and the $\mathrm{T} 2$ findings usually permit the diagnosis, since these pseudo-lesions are invisible on that sequence.

Potential pitfalls Areas of focal fat sparing are enhanced because they have functional hepatocytes and these can appear as real focal lesions in the dynamic phases. However, the other imaging findings described permit the diagnosis.

\section{Intrahepatic cholangiocarcinoma}

Overview Cholangiocarcinoma is the most common biliary tumour and the second most common primary malignant hepatic tumour in adults. Risk factors for the development of cholangiocarcinoma include primary sclerosing cholangitis (PSC), familial polyposis, choledochal cyst, biliary papillomatosis and clonorchiasis.

Unenhanced MRI features Intrahepatic cholangiocarcinoma may be mass forming or peribiliar infiltrative. They are usually hypointense on T1-weighted MR images and hyperintense on
T2-weighted images. Associated findings may include adjacent biliary ductal dilatation, capsular retraction, satellite nodules, vascular encasement and hepatolithiasis [32-34].

Dynamic imaging with extracellular contrast agent On contrast-enhanced images, it demonstrates initial peripheral enhancement with concentric internal filling on delayedphase images.

Imaging with $G d-E O B-D T P A$ Usually no enhancement is seen in the hepatocellular phase due to lack of hepatocytes (Fig. 18).

Differential diagnosis $\mathrm{HCC}$ and cholangitis. The delayed enhancement and the typical hilar location point to cholangiocarcinoma.

Potential pitfalls Delayed enhancement of cholangiocarcinoma is one of the characteristic findings when using extracellular agents, due to its abundant fibrosis. However, the tumour shows low signal intensity on delayed and hepatobiliary phases of Gd-EOB-DTPA, due to strong enhancement of the surrounding liver parenchyma.

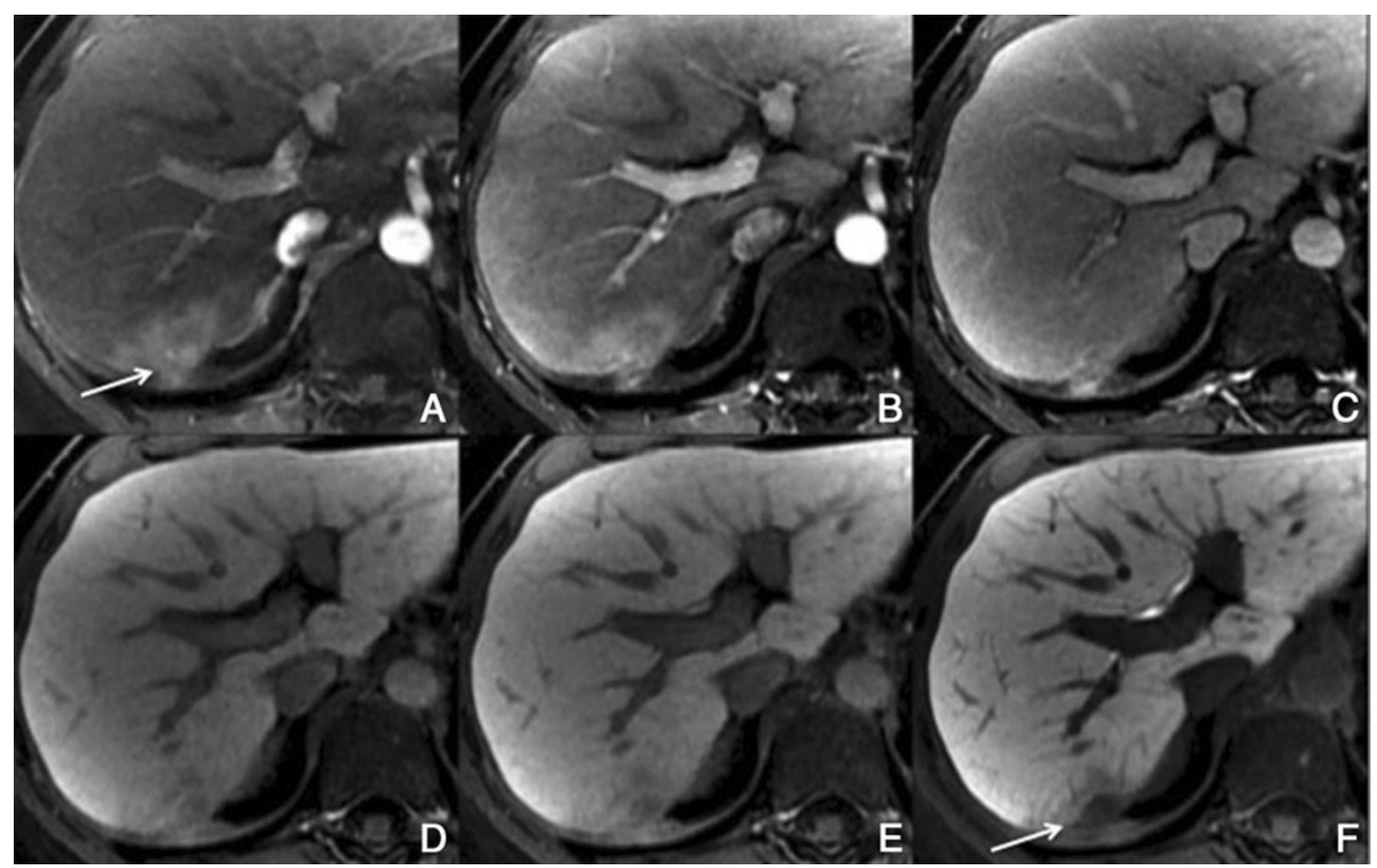

Fig. 21 Foreign body reaction, in a 41-year-old man that suffered a car accident after a New Year's dinner. a, b Arterial phase, c PVP, d 5 min, e $8 \mathrm{~min}, \mathbf{f ~ H C P}$. In the posterior aspect of the left lobe we see a fluffy heterogeneous hyperenhancing area, that progressively washes out and gives origin to a Gd-EOB-DTPA defect (thin arrows). Histologically proven, after surgery, to be an inflammatory reaction to a small carrot fragment 


\section{Hypervascular metastases}

Overview Islet cell tumours, breast cancer, melanoma, thyroid cancer and carcinoid tumour are among the most common primary tumours that lead to hypervascular hepatic metastases.

Unenhanced MRI features These metastatic lesions usually demonstrate low signal intensity on T1-weighted MR images and are iso to hyperintense on T2-weighted images.

Dynamic imaging with extracellular contrast agent Hypervascular metastases are best seen during the arterial phase of enhancement. Small hypervascular metastases usually present with homogeneous enhancement, whereas larger lesions appear heterogeneous or show an enhancing peripheral rim surrounding the necrosis. Frequently, centripetal contrast enhancement is observed, as well as irregular or peripheral washout. Hypervascular metastatic lesions may show a target appearance on delayed phase, with the rim appearing hypointense relative to the centre. This centripetal progression of enhancement, with simultaneous peripheral washout, is specific for malignancy.

Imaging with $G d-E O B-D T P A$ Generally no enhancement is seen in the hepatocellular phase.

Differential diagnosis HCC, THID, adenoma and FNH. The presence of multiple lesions in a non-cirrhotic liver, in a patient with a known malignancy and a defect on the HCP point to metastases.

Potential pitfalls In the centre of metastatic lesions, there may be pooling of Gd-EOB-DTPA in the extracellular matrix of the lesion, giving the misguiding appearance of uptake in the hepatobiliary phase (Fig. 19).

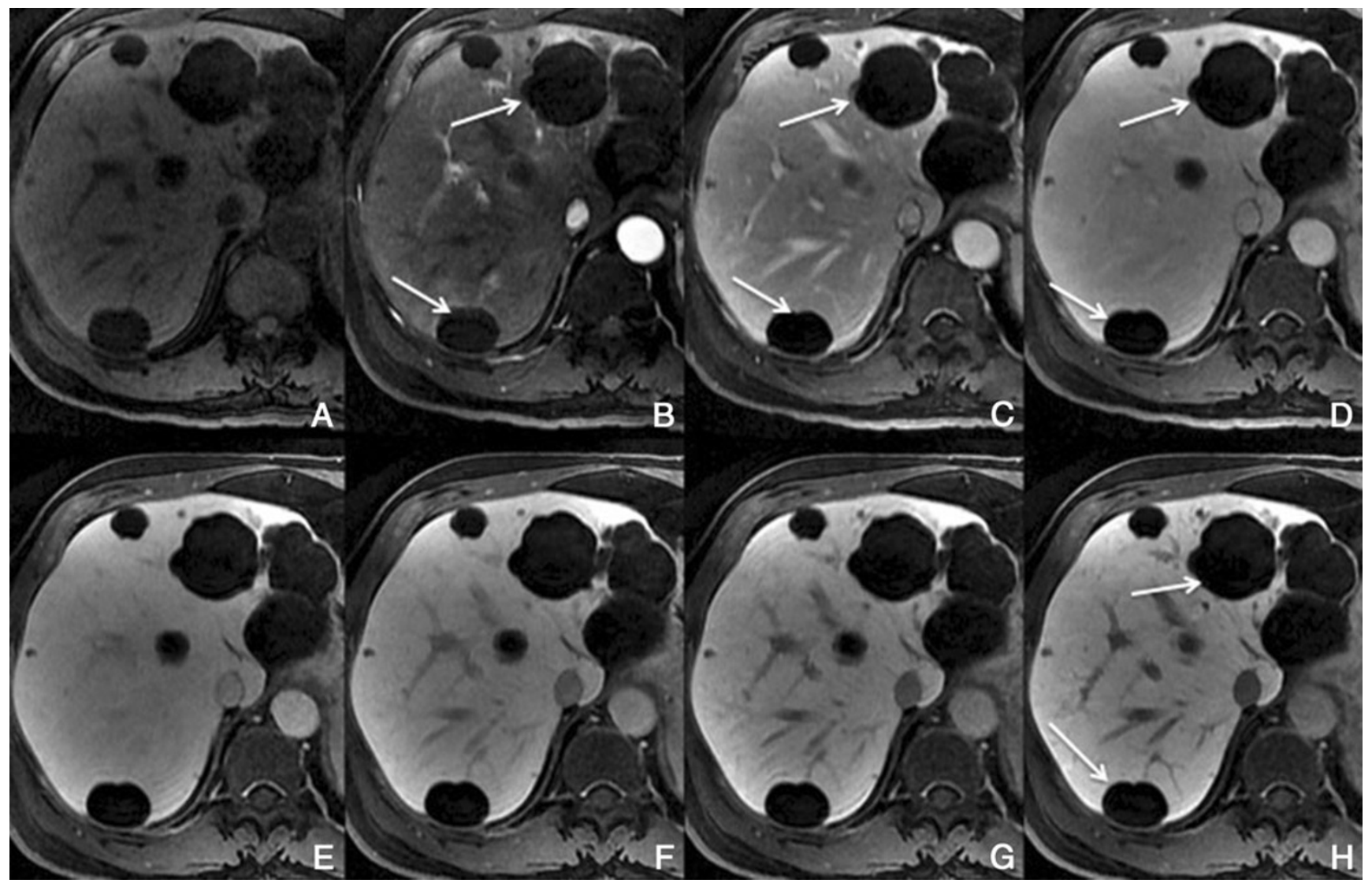

Fig. 22 Multiple hepatic cysts, in a 47-year-old woman, with no liver disease. a Pre-contrast, b arterial, c PVP, d $3 \mathrm{~min}$, e $5 \mathrm{~min}, \mathbf{f} 8 \mathrm{~min}, \mathbf{g}$ $10 \mathrm{~min}, \mathbf{h}$ HCP. Multiple rounded hypointense lesions (arrows), with no enhancement during all the phases. These lesions were also markedly hyperintense in $\mathrm{T} 2$-weighted imaging (not shown) 
Fig. 23 Angiomyolipoma in a 40-year-old man with a history of renal lesions. a Arterial phase, b T2 fat sat, c OP, d IP. Large mass-forming lesion, irregular contour, with mild heterogeneous enhancement in the arterial phase (arrow). It loses internal signal in $\mathrm{OP}$, indicating the presence of intracellular fat (black arrow). These were histologically proven to be an angiomyolipoma
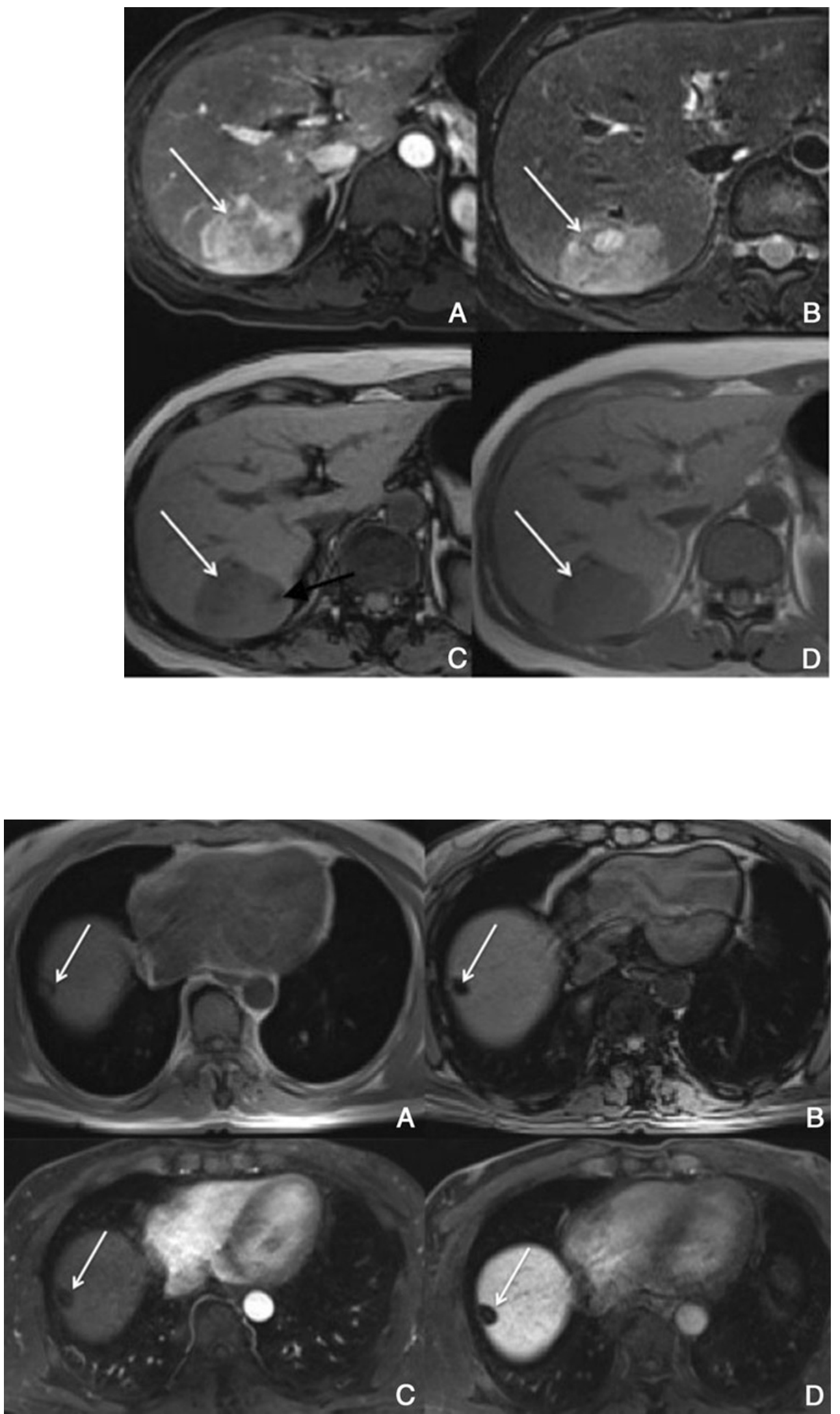

Fig. 24 Pseudolipoma

(Glisson's capsule lipoma). a IP b OP $\mathbf{c}$ arterial d HCP. Small, no enhancing peripheral lesion (arrow), losing internal signal in $\mathrm{OP}$, indicating the presence of intracellular fat.

Histologically proven, after surgery, to be a pseudolipoma 


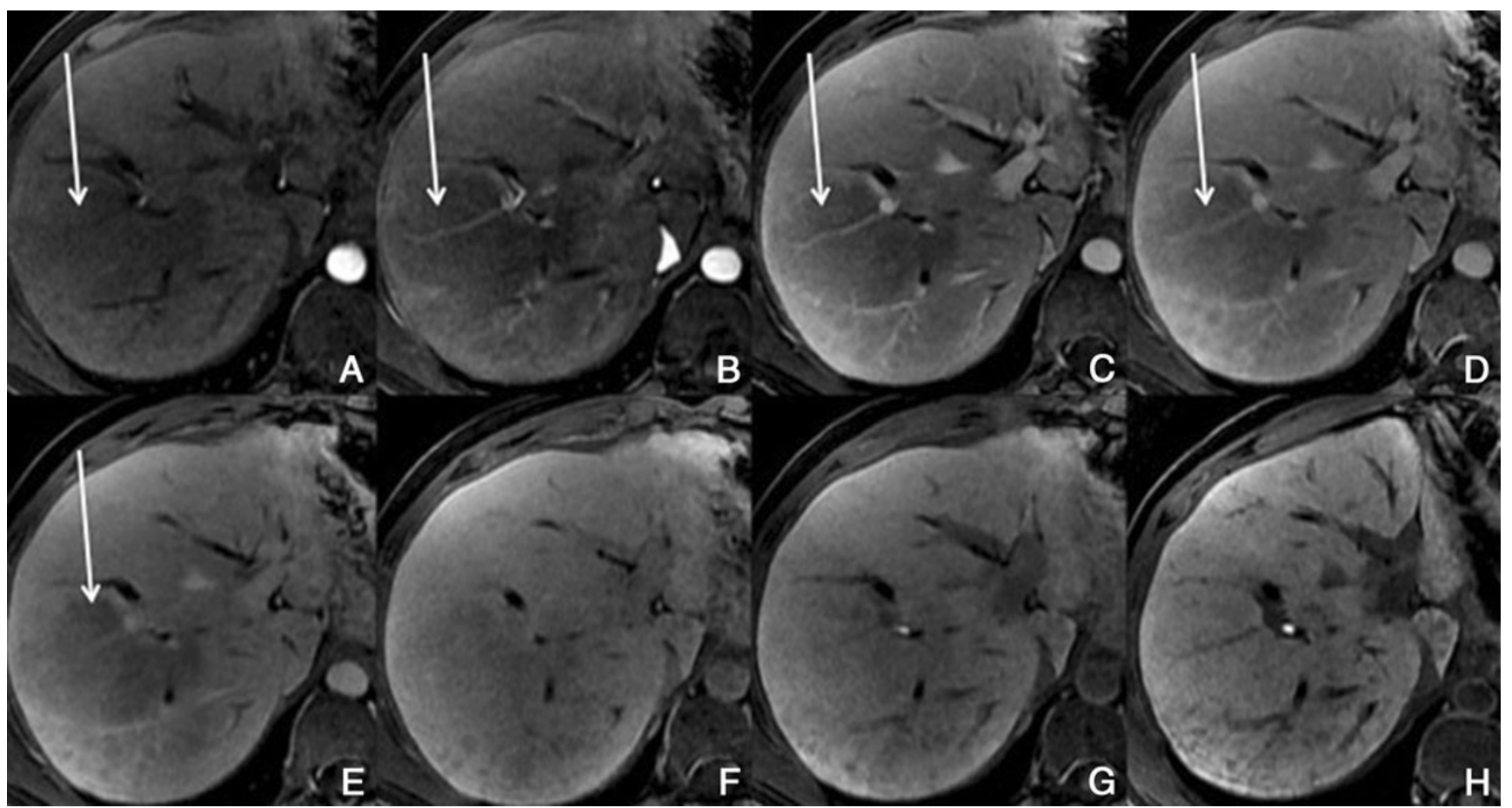

Fig. 25 Inflammatory pseudotumour, in a 52-year-old man with PSC. a, b Arterial phase, c PVP, d 3 min, e 5 min, f HCP. Ill-defined hypointense area, in the right lobe (arrows), seen in the dynamic phases, not visualised in the HCP, proven to represent an inflammatory pseudolesion

Fig. 26 TP granulomas, in a 42-year-old man with active pulmonary TP. a Arterial phase, b PVP, c delayed phase, $\mathbf{d}$ HCP. There are two nodular lesions (arrows), with faint peripheral enhancement, that appear as defects in the HCP, as they have no hepatocytes to uptake GdEOB-DTPA

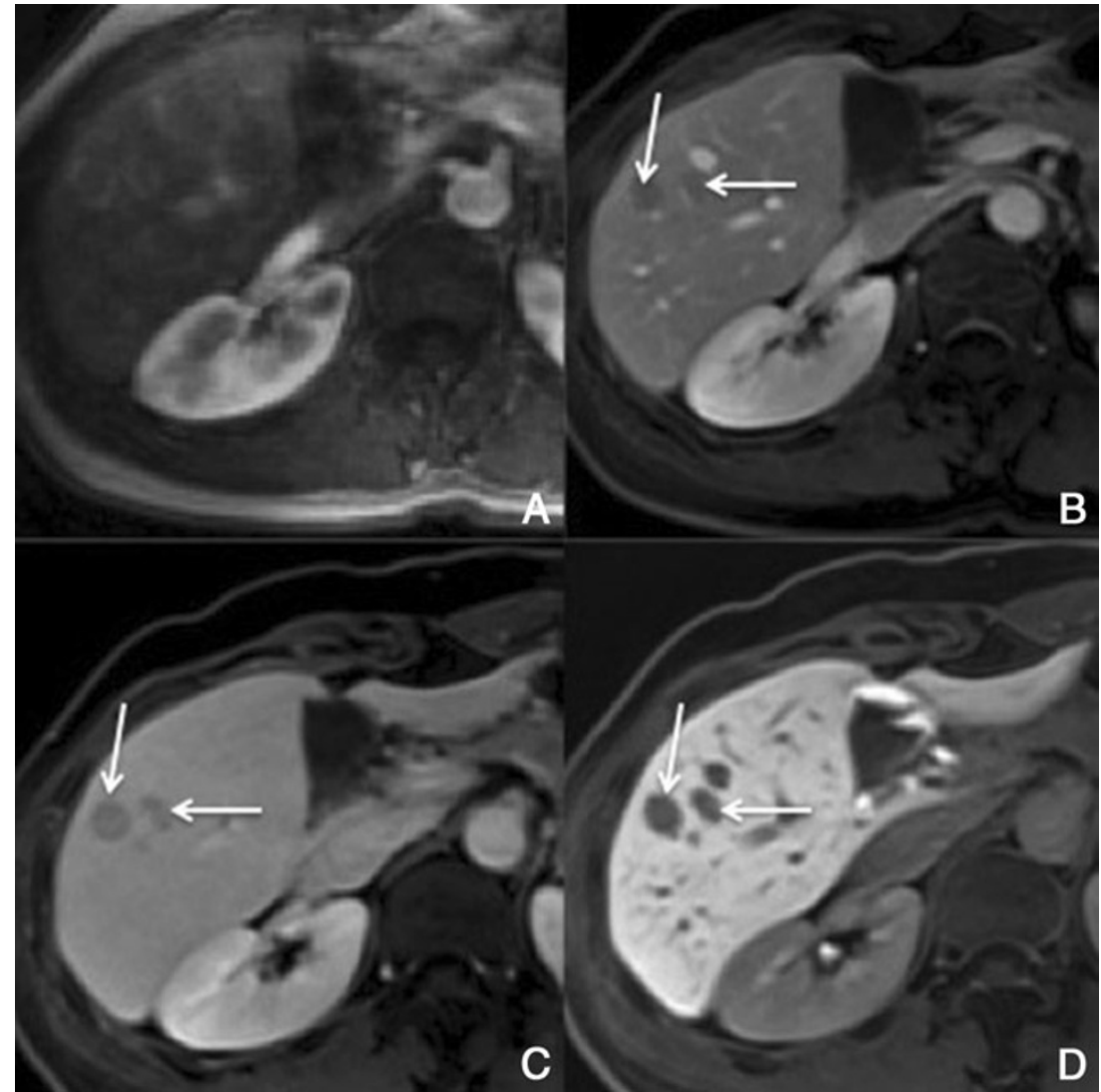




\section{Hypovascular metastases}

Overview Colon, lung, prostate, gastric and transitional cell carcinomas are the most common primary tumours with hypovascular metastases to the liver.

Unenhanced MRI features These metastatic lesions usually demonstrate low signal intensity on T1-weighted MR images and are iso to hyperintense on $\mathrm{T} 2$-weighted images.

Dynamic imaging with extracellular contrast agent The ring-like enhancement surrounding liver metastases is typically seen during the hepatic arterial phase. In the portal and delayed phase, the metastases often show washout in the outer parts and a progressive enhancement toward the centre of the lesions (Fig. 20).

Imaging with Gd-EOB-DTPA No enhancement is seen in the hepatocellular phase.
Differential diagnosis HCC, cholangiocarcinoma and focal eosinophilic infiltration. Multiple lesions, a known primary malignant tumour and a non-cirrhotic liver, point to the diagnosis. The pattern of enhancement and the spherical shape are other helpful findings.

Potential pitfalls As mentioned earlier, with Gd-EOBDTPA, the differential diagnosis can be difficult.

\section{Foreign body reaction}

Overview Various foreign bodies introduced into the human organism during surgery or trauma, as well as exposure to some chemical substances, may cause a granulomatous reaction.

Unenhanced MRI features Imaging features are usually hypointensity in T1-weighted images and hyperintensity in T2-weighted images, with possible hypointense bands within the cystic cavity.

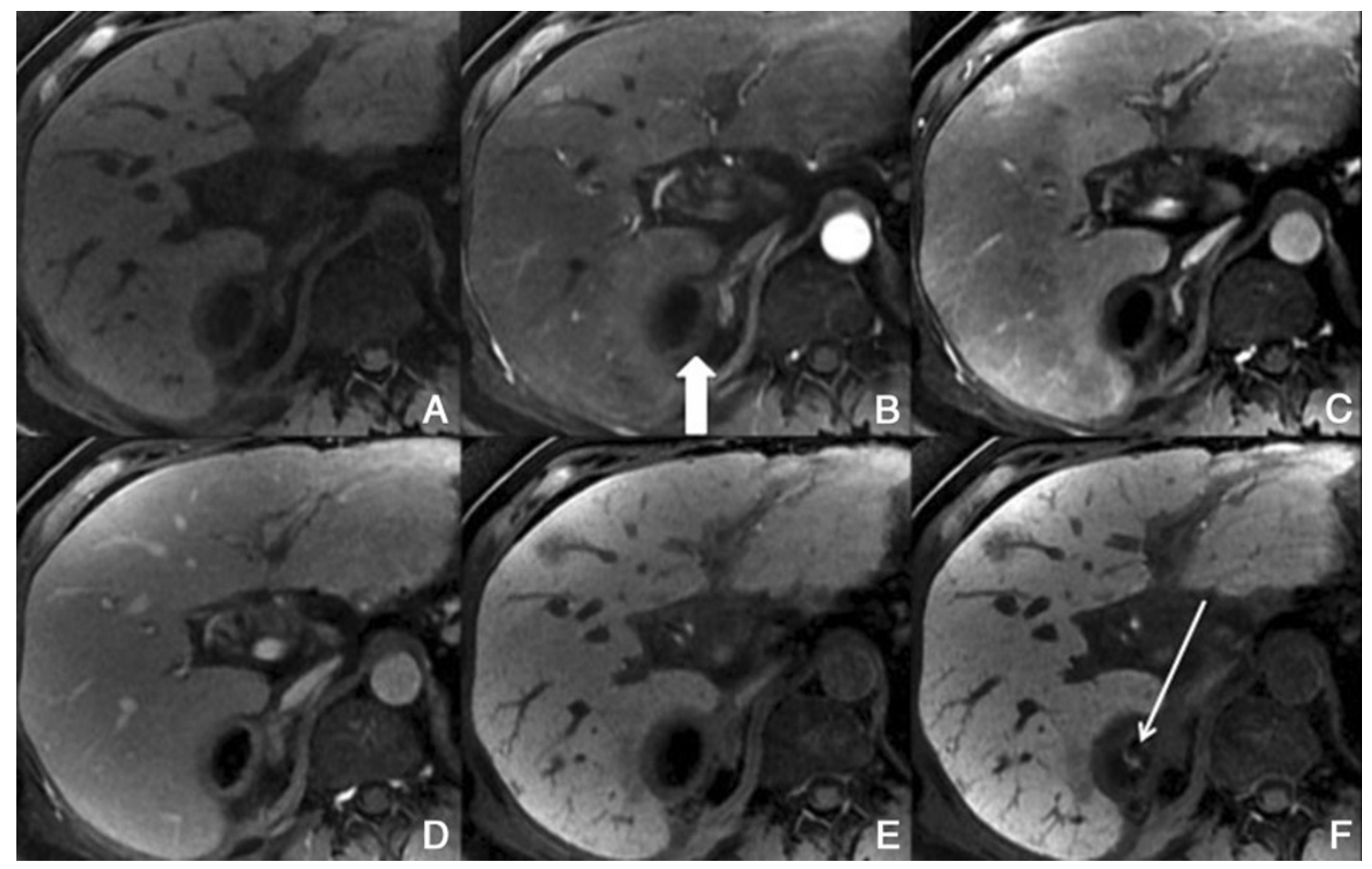

Fig. 27 Liver abscess, in a 54-year-old man with history of choledocal litiasis. a Pre-contrast, b, c arterial, d $3 \mathrm{~min}$, e $5 \mathrm{~min}$, f HCP. On the posterior aspect of the liver, there is a thick-walled hypointense lesion (solid arrow), with no internal enhancement during the dynamic phases. Note the excretion of Gd-EOB-DTPA on the HCP, proving the connection of this abscess with the biliary tree 
Fig. 28 Liver Lymphoma, in a 39-year-old woman. a Precontrast, $\mathbf{b}$ arterial phase, $\mathbf{c}$ PVP, d HCP. Large infiltrative lesion in the right lobe (arrows), hypointense in the pre-contrast phase, with faint heterogeneous arterial enhancement, with washout in the PVP, not taking up Gd-EOB-DTPA in the HCP. Note involvement of the tumour along the periportal route (black arrow) and right adrenal gland (white arrows)

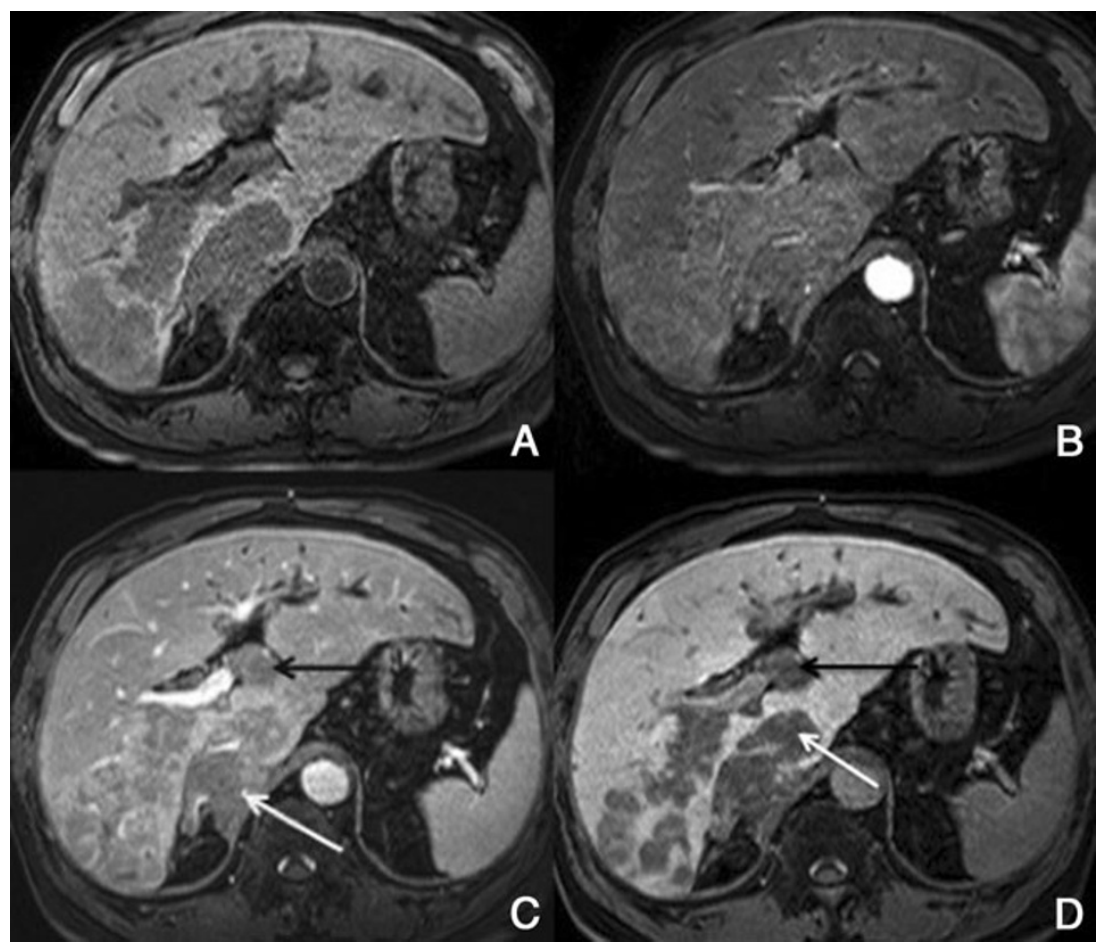

Dynamic imaging with extracellular contrast agent: It shows peripheral enhancement of the mass on arterial or portal venous phase.

Imaging with Gd-EOB-DTPA No enhancement is seen in the hepatocellular phase (Fig. 21).

Differential diagnosis Metastatic disease. It could be considered in patients with a perihepatic lesion with previous history of trauma or surgery.

Potential pitfalls A foreign body can gradually move from a subcapsular to an intraparenchymal position.

Lesions in which the lesion behaviour is equivalent to that of when using an ECF contrast agent

Focal hepatic lesions that show similar behaviour with ECF contrast agent and Gd-EOB-DTPA include hepatic cysts (Fig. 22), biliary hamartomas, biliary cystadenoma or carcinomas, angiomyolipoma (Fig. 23), lipoma (Fig. 24), inflammatory pseudotumour (Fig. 25), granulomatous disease (Fig. 26), hepatic abscesses (Fig. 27) and lymphoma (Fig. 28).
Lesions in which the authors have no experience

The following lesions were never studied with Gd-EOBDTPA-enhanced MRI: hematoma, peliosis, paranglioma and angiosarcoma.

\section{Summary}

For a summary, see Tables 1 and 2.

Table 1 Patterns of enhancement and behaviour

\begin{tabular}{lllll}
\hline Lesion & $\begin{array}{l}\text { Arterial } \\
\text { phase }\end{array}$ & $\begin{array}{l}\text { PV } \\
\text { phase }\end{array}$ & $\begin{array}{l}\text { Delayed } \\
\text { phase }\end{array}$ & $\begin{array}{l}\text { Hepatobiliary } \\
\text { phase }\end{array}$ \\
\hline Benign & $\Rightarrow$ & $\Rightarrow$ & $\Rightarrow$ & $\Rightarrow$ \\
Benign & $\Uparrow$ & $\Rightarrow$ & $\Rightarrow$ & $\Rightarrow$ \\
Benign & $\Uparrow$ & $\Downarrow$ & $\Downarrow$ & $\Uparrow$ \\
Indeterminate & $\Downarrow$ & $\Downarrow$ & $\Downarrow$ & $\Downarrow$ or $\Uparrow$ \\
Malignant & $\Uparrow$ & $\Downarrow$ & $\Downarrow$ & $\Downarrow$ \\
Malignant & $\Downarrow$ & $\Uparrow$ & $\Downarrow$ & $\Downarrow$ \\
\hline
\end{tabular}




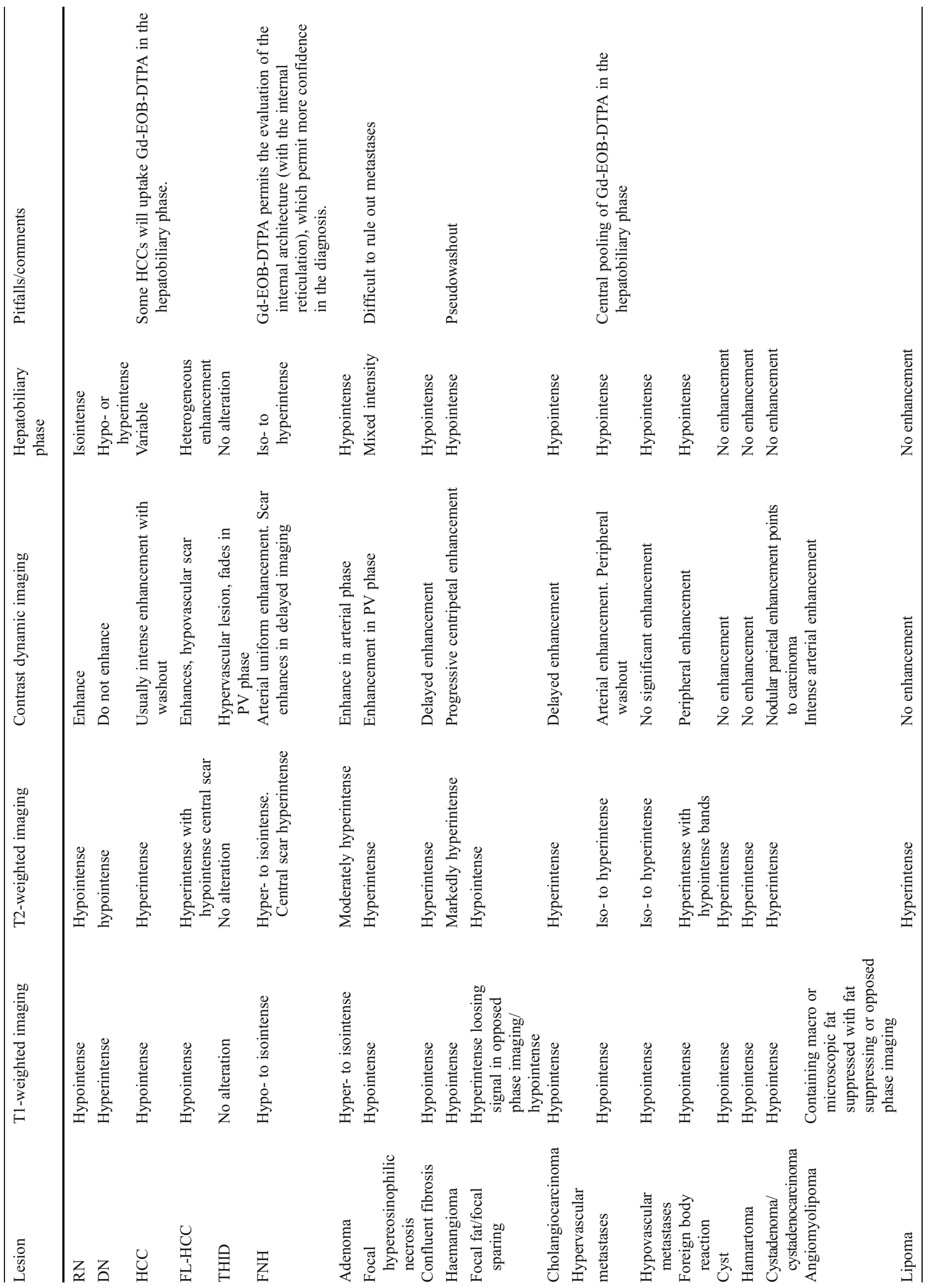


Open Access This article is distributed under the terms of the Creative Commons Attribution License which permits any use, distribution, and reproduction in any medium, provided the original author(s) and the source are credited.

\section{References}

1. Nam K, Suk K, Jun W, Suk HL, Dae H, Gwang HK, Hyung Il S (2009) Biliary MR imaging with Gd-EOB-DTPA and its clinical applications. Radiographics 29:1707-1724

2. Reimer P, Schneider G, Schima W (2004) Hepatobiliary contrast agents for contrast-enhanced MRI of the liver: properties, clinical development and applications. Eur Radiol 14:559-578

3. Tsuboyama T, Onishi H, Kim T, Akita H, Hori M, Tatsumi M, Nakamoto A, Nagano H, Matsuura N, Wakasa K, Tomoda K (2010) Hepatocellular carcinoma: hepatocyte-selective enhancement at gadoxetic acid-enhanced MR imaging - correlation with expression of sinusoidal and canalicular transporters and bile accumulation. Radiology 255(3):824-833

4. Kitao A, Zen Y, Matsui O, Gabata T, Kobayashi S, Koda W, Kozaka K, Yoneda N, Yamashita T, Kaneko S, Nakanuma Y (2010) Hepatocellular carcinoma: signal intensity at gadoxetic acid- enhanced MR imaging - correlation with molecular transporters and histopathologic features. Radiology 256(3):817-826

5. Seale MK, Catalano OA, Saini S, Hahn PF, Sahani DV (2009) Hepatobiliary-specific MR contrast agents: role in imaging the liver and biliary tree. Radiographics 29:1725-1748

6. Gandhi SN, Brown MA, Wong JG, Aguirre DA, Sirlin CB (2006) MR contrast agents for liver imaging: what, when, how. Radiographics 26:1621-1636

7. Sun HY, Lee JM, Shin CI, Lee DH, Moon SK, Kim KW, Han JK, Choi BI (2010) Gadoxetic acid-enhanced magnetic resonance imaging for differentiating small hepatocellular carcinomas $(2 \mathrm{~cm}$ in diameter) from arterial enhancing pseudolesions special emphasis on hepatobiliary phase imaging. Investig Radiol 45(2):96-103

8. Zech CJ, Grazioli L, Breuer J, Reiser M, Schoenberg SO (2008) Diagnostic performance and description of morphological features of focal nodular hyperplasia in Gd-EOB-DTPA-enhanced liver magnetic resonance imaging: results of a multicenter trial. Investig Radiol 43(7):504-511

9. Tajima T, Honda H, Taguchi K et al (2002) Sequential hemodynamic change in hepatocellular carcinoma and dysplastic nodules: CT angiography and pathologic correlation. AJR Am J Roentgenol 178:885-897

10. Roncalli M, Roz E, Coggi G et al (1999) The vascular profile of regenerative and dysplastic nodules of the cirrhotic liver: implications for diagnosis and classification. Hepatology 30:1174-1178

11. Cruite I, Schroeder M, Merkle EM, Sirlin CB (2010) Gadoxetate disodium-enhanced MRI of the liver: part 2, protocol optimization and lesion appearance in the cirrhotic liver. AJR Am J Roentgenol 195:29-41

12. Choi JY, Lee HC, Yim JH, Shim JH, Lim HS, Shin YM, Yu ES, Suh DJ (2011) Focal nodular hyperplasia or focal nodular hyperplasia-like lesions of the liver: a special emphasis on diagnosis. J Gastroenterol Hepatol 26(6):1004-1009

13. Libbrecht L, Cassiman D, Verslype C, Maleux G, Van Hees D, Pirenne J, Nevens F, Roskams T (2006) Clinicopathological features of focal nodular hyperplasia-like nodules in 130 cirrhotic explant livers. Am J Gastroenterol 101:2341-2346

14. Choi BI, Takayasu K, Han MC (1993) Small hepatocellular carcinoma and associated lesions of the liver: pathology, pathogenesis, imaging findings. AJR Am J Roentgenol 160:1177-1187

15. Levy AD (2002) Malignant liver tumours. Clin Liver Dis 6:147-164 
16. Hanna RF, Aguirre DA, Kased N, Emery SC, Peterson MR, Sirlin CB (2008) Cirrhosis-associated hepatocellular nodules: correlation of histopathologic and MR imaging features. Radiographics 28:747-769

17. Huppertz A, Haraida S, Kraus A et al (2005) Enhancement of focal liver lesions at gadoxetic acid-enhanced MR imaging: correlation with histopathologic findings and spiral CT-initial observations. Radiology 234:468-478

18. Kim SH (2009) Gadoxetic acid-enhanced MRI versus triple-phase MDCT for the preoperative detection of hepatocellular carcinoma. AJR Am J Roentgenol 192:1675-1681

19. Fujita M, Yamamoto R, Fritz-Zieroth (1996) Contrast enhancement with Gd-EOB-DTPA in MR imaging of hepatocellular carcinoma in mice: a comparison with superparamagnetic iron oxide. J Magn Reson Imaging 6(3):472-477

20. Shimofusa R (2009) Magnetic resonance imaging of hepatocellular carcinoma: a pictorial review of novel insights into pathophysiological features revealed by magnetic resonance imaging. J Hepatobiliary Pancreat Surg 17(5):583-589

21. Ringe KI, Husarik DB, Sirlin CB, Merkle EM (2010) Gadoxetate disodium-enhanced MRI of the liver: part 1, protocol optimization and lesion appearance in the noncirrhotic liver. AJR Am J Roentgenol 195(1):13-28

22. Ahn JH, Yu JS, Hwang SW, Chung JJ, Kim JH, Kim KW (2010) Nontumourous arterioportal shunts in the liver: CT and MRI findings considering mechanisms and fate. Eur Radiol 20:385-394

23. Motosugi U, Ichikawa T, Sou H, Sano K, Tominaga L, Muhi A, Araki T (2010) Distinguishing hypervascular pseudolesions of the liver from hypervascular hepatocellular carcinomas with gadoxetic acid-enhanced MR imaging. Radiology 256(1):151-158

24. Wanless IR, Mawdsley C, Adams R (1985) On the pathogenesis of focal nodular hyperplasia of the liver. Hepatology 5(6):1194-2000
25. Kamel IR, Liapi E, Fishman EK (2006) Focal nodular hyperplasia: lesion evaluation using 16-MDCT and 3D CT angiography. AJR Am J Roentgenol 186(6):1587-1596

26. Grazioli L, Morana G, Federle MP et al (2001) Focal nodular hyperplasia: morphologic and functional information from MR imaging with gadobenate dimeglumine. Radiology 221:731-739

27. Brancatelli G, Federle MP, Vullierme MP, Lagalla R, Midiri M, Vilgrain V (2006) CT and MR imaging evaluation of hepatic adenoma. J Comput Assist Tomogr 30(5):745-750

28. Ichikawa T, Federle MP, Grazioli L, Nalesnik M (2000) Hepatocellular adenoma: multiphasic CT and histopathologic findings in 25 patients. Radiology 214(3):861-868

29. Hur J, Park M, Yu J, Lim J, Hong SW, Kim KW (2005) Focal eosinophilic necrosis versus metastasis in the liver: the usefulness of two-phase dynamic CT. AJR Am J Roentgenol 184:1541-1548

30. Kim Y, Kim C, Moon W, Cho B, Lee, Lee JM (2005) MRI findings of focal eosinophilic liver diseases. AJR Am J Roentgenol 184:1541-1548

31. Doo KW, Lee CH, Choi JW, Lee J, Kim KA, Park CM (2009) "Pseudo washout" sign in high-flow hepatic hemangioma on gadoxetic acid contrast-enhanced MRI mimicking hypervascular tumour. AJR Am J Roentgenol 193:W490-W496

32. Anderson SW, Kruskal JB, Kane RA (2009) Benign hepatic tumours and iatrogenic pseudotumours. Radiographics 29:211-229

33. Semelka RC, Martin DR, Balci C, Lance T (2001) Focal liver lesions: comparison of dual-phase CT and multisequence multiplanar MR imaging including dynamic gadolinium enhancement. J Magn Reson Imaging 13(3):397-401

34. Hussain SM, Zondervan PE, IJzermans JN, Schalm SW, Man RA, Krestin GP (2002) Benign versus malignant hepatic nodules: MR imaging findings with pathologic correlation. Radiographics 22:1023-1039 